\title{
Sliding Mode Heading Control of an Over-Actuated, Hover-Capable Autonomous Underwater Vehicle with Experimental Verification
}

\author{
Kantapon Tanakitkorn \\ Fluid Structure Interactions Research Group \\ University of Southampton \\ Southampton, UK \\ kantapon.imc@gmail.com \\ Stephen R. Turnock \\ Fluid Structure Interactions Research Group \\ University of Southampton \\ Southampton, UK \\ S.R.Turnock@soton.ac.uk
}

\author{
Philip A. Wilson \\ Fluid Structure Interactions Research Group \\ University of Southampton \\ Southampton, UK \\ Philip.Wilson@soton.ac.uk \\ Alexander B. Phillips \\ Marine Autonomous Robotic Systems \\ National Oceanography Centre \\ Southampton, UK \\ abp@noc.ac.uk
}

\begin{abstract}
A sliding mode heading control system is developed for over-actuated, hover-capable AUVs operating over a range of forward speeds. A simplified switching function is introduced, and simulation studies are proposed accordingly. The results with this novel switching function show a significant improvement in the chattering problem when compared to other conventional switching function candidates. Studies on sensitivities to a range of hydrodynamic parameter uncertainties are presented, and the parameters that have major influences on the sliding mode control performance are highlighted. The proposed control system is also proven in the field trials to enhance vehicle response, yielding consistent and robust performance over the entire range of vehicle's speeds, even when subjected to external disturbance. It also shows superior heading tracking performance when compared to a P-D approach implemented on the same vehicle. However, this improved performance requires intense control actions as a trade-off, causing the AUV to expend more energy than with the P-D approach. Path following trials, where the AUV is demanded to follow a set of GPS coordinates at the water surface, are presented. These demonstrate the applicability of the proposed heading control system in a practical operation. The presented SMC scheme can be applied to any vehicle straightforwardly, but the control allocation part may need to be modified regarding the actuator configuration of that vehicle.
\end{abstract}

\section{Introduction}

Autonomous underwater vehicles (AUVs) are typically designed with an emphasis on minimising total resistance to suit long-range flight-style operation - they are of a torpedo-shaped body that is equipped with a main propeller and control surfaces (Allen et al., 1997; McPhail, 2009). These conventional AUVs, however, are not able to carry out detailed inspection tasks at zero or slow forward speeds since the control surfaces become ineffective in this regime (Burcher and Rydill, 1995, p.169). 
Various concepts for next generation of hover-capable AUVs have been developed to overcome such a limitation (Marco and Healey, 2000; Dunbabin et al., 2005; Packard et al., 2010; Ribas et al., 2015). Typically, thrusters are added to the design to provide additional control forces for low-speed operation. See Delphin2 AUV in figure 1 for example. Since this AUV can be considered to have a redundant set of actuators for controlling a given degree of freedom, it is referred to as over-actuated AUV. More details on the Delphin2 AUV development are given by (Liu et al., 2009; Steenson et al., 2011a,b; Phillips et al., 2013; Steenson et al., 2014; Tanakitkorn et al., 2016).

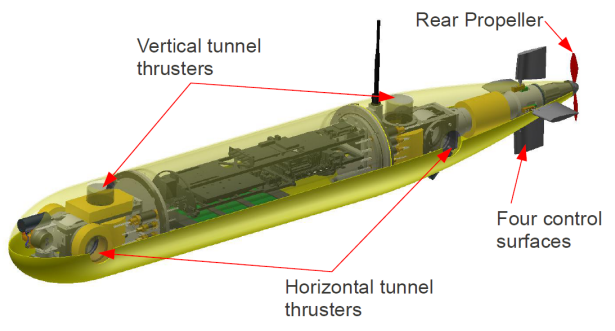

Figure 1: Delphin2 AUV with an over-actuated configuration (Steenson, 2013).

Different heading control strategies are required for an over-actuated AUV operating in difference circumstances. Typically, rudders are ideal for high-speed operation because they are both energy efficient and effective in this regime. On the other hand, extra forces from horizontal thrusters are required when operating in the low-speed regime, where the control surfaces become ineffective, or when dealing with large heading errors, and rudders alone cannot make the vehicle turns quickly enough.

The heading control problem may be split into two cascade modules (Fossen and Johansen, 2006; Kokegei et al., 2011): the first module, a control law, determines a generalised moment that is required for heading tracking; the second module, a control allocation, allocates the generalised moment between thrusters and rudders.

The control allocation problem may be solved using a daisy-chaining approach where actuators are rearranged in a hierarchy based on their effectiveness and efficiency (Oppenheimer et al., 2006). The control demand is first allocated on the top actuator in the hierarchy until reaching the actuator limit; the residual control force is then allocated on the next actuators in the hierarchy. This concept is also implemented by Kokegei et al. (2011) under the name 2-state control. Alternatively, the control allocation problem may be solved algebraically using a pseudo-inverse technique (Jin et al., 2014), or formulated as an optimisation problem using, e.g., quadratic programming (QP) approach (Hanger et al., 2011).

The control law may be derived using sliding mode control (SMC) techniques (Perruquetti and Barbot, 2002; Morari, 2012; Shtessel et al., 2014). A sliding variable is designed to represent the desired response. A discrete switching term, also known as a relay control law, is devised using the sliding variable to drive the system back to the equilibrium by gliding along the sliding surface, where the sliding variable is zero. Continuous stabilising terms may be included as an addition to the control law to improve the reaching phase performance. This term may be designed based on a proportional-derivative (PD) control (Ouyang et al., 2015), or state feedback control, where the gains are obtained using a pole-placement technique (Utkin, 1977; Vuilmet, 2005); linear quadratic regulator (LQR) technique may also be included in the continuous stabilising terms (Jantapremjit and Wilson, 2007). Furthermore, an integral term may be added to a sliding variable to eliminate a steady-state error due to external disturbances and parameter uncertainties (Shi et al., 2008; Kim et al., 2015).

A number of SMC applications on AUVs have been reported in the literature. Lea (1998) has proposed a SMC based autopilot for decoupled heading, speed and depth control for Subzero II AUV. The results show superior performance when compared to a proportional-integral-derivative (PID) approach implemented on the same vehicle. Jantapremjit and Wilson (2008) have employed SMC scheme for a 3D path following 
application. Lekkas (2014) has proposed SMC heading control based on a nonlinear extension of Nomoto's 1st-order model.

Typically, an SMC approach with a discontinuous switching function is prone to a "chattering phenomenon" (Perruquetti and Barbot, 2002). It is the event where the system exhibits a high-frequency oscillation (see figure 2). This oscillation could lead to high actuator wear, excessive energy lost or even causes the system to become unstable. A range of approaches have been developed to improve the chattering problem. Slotine and $\mathrm{Li}$ (1991) have shown that the chattering is attenuated when a thin boundary layer is applied to a sliding surface. This boundary layer may be used in conjunction with a continuous approximation of the switching function, e.g., hyperbolic tangent function, producing much less noisy response (Vuilmet, 2005; Conte et al., 2016). Higher-order sliding mode control (HOSMC) approaches can also be used for chattering alleviation (Vuilmet, 2005; Levant, 2007).

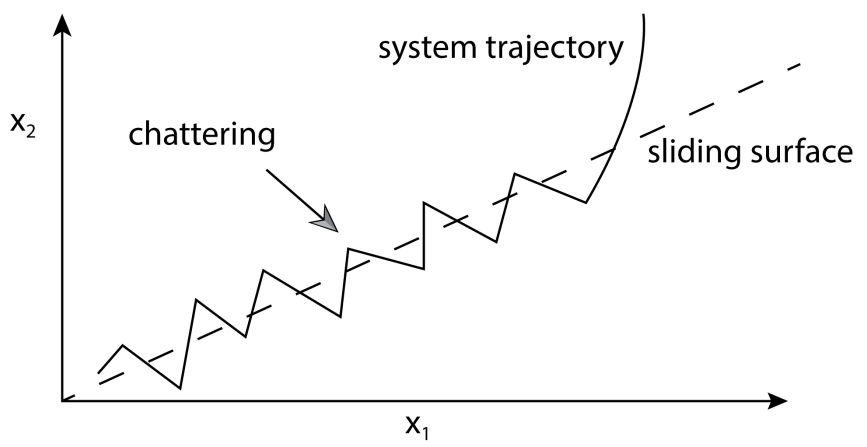

Figure 2: An example of phase plot showing a chattering phenomenon.

Some alternative control techniques have successfully been applied to the heading control problem for the AUV. Naeem et al. (2005) has presented a heading control system for a hammerhead AUV designed using a model predictive control (MPC) approach where a genetic algorithm (GA) is used as a minimiser. This approach is computationally expensive and could not be used at the high sample rate. A gain scheduled static output feedback controller is proposed by Silvestre and Pascoal (2004). In this work, multiple sets of gains are derived for many difference forward speeds and are scheduled accordingly. Yang et al. (2015) has present a development of $H_{\infty}$ heading control. The results are compared with those obtained with a PID control technique, showing superior performance as well as robustness against parameter uncertainties. Li and $\mathrm{Su}$ (2016) have presented a heading control system for the REMUS AUV based on backstepping and Lyapunov redesigned technique. This is demonstrated by a simulation, showing a possibility of using both a tunnel thruster and a moving mass to enhance the vehicle performance in a low-speed regime.

This paper focuses on design, simulation and experimental testing of sliding mode heading control system for an over-actuated AUV operating over a range of forward speeds. A simplified switching function is introduced to alleviate the chattering phenomenon. A control allocation technique is employed to effectively distribute the control demand between the thrusters and the rudders. The proposed control system is experimentally verified in the lake trials on the Delphin2 AUV and benchmarked against the simple P-D control approach implemented on the same vehicle. The control performance is also demonstrated by path following missions, where the controller collaborates with the guidance module in the AUV's system.

The rest of the paper is organised in the following order. In section 2, the horizontal-plane dynamics model of Delphin2 is presented. The sliding mode control system is designed in section 3. A few choices of switching functions for a chattering alleviation are presented in section 4 . Section 5 provides simulation studies, focusing on choices of switching functions as well as sensitivities to the model parameters. An experimental setup is explained in section 6 , following with experimental results and discussion in section 7 . Conclusions are given in section 8 . 


\section{Vehicle dynamics model}

A dynamics model of the AUV may be used to estimate the vehicle response in difference situations. This is particularly important for when developing a non-linear control system. A horizontal-plane dynamics model may be described in a matrix form according to Fossen (2011):

$$
\boldsymbol{M} \cdot \dot{\nu}+\boldsymbol{C}(\nu) \cdot \nu+\boldsymbol{D}(\nu) \cdot \nu=\tau,
$$

where $\nu=[u, v, r]^{T}$ is the velocity vector, $\eta=[x, y, \psi]^{T}$ is a pose vector, and $\tau=[X, Y, N]^{T}$ is a generalised force vector due to actuators. The inertia matrix, Coriolis-centripetal matrix and damping matrix for decoupled surge dynamics are:

$$
\begin{gathered}
M=\left[\begin{array}{ccc}
m-X_{\dot{u}} & 0 & 0 \\
0 & m-Y_{\dot{v}} & 0 \\
0 & 0 & I_{z z}-N_{\dot{r}}
\end{array}\right], \\
\boldsymbol{C}(\nu)=\left[\begin{array}{ccc}
0 & 0 & -m\left(x_{g} r+v\right)+Y_{\dot{v}} v \\
0 & 0 & -X_{\dot{u}} u+m u \\
m\left(x_{g} r+v\right)-Y_{\dot{v}} v & X_{\dot{u}} u-m u & 0
\end{array}\right], \\
\boldsymbol{D}(\nu)=-\left[\begin{array}{ccc}
X_{u}+X_{|u| u \mid}|u| & 0 & 0 \\
0 & Y_{v}+Y_{|v| v|v|} & Y_{r}+Y_{|r| r \mid}|r| \\
0 & N_{v}+N_{|v| v|v|} & N_{r}+N_{|r| r \mid}|r|
\end{array}\right] .
\end{gathered}
$$

Since the Delphin2 AUV is almost symmetric about the longitudinal axis, the dynamics model parameters on this horizontal-plane subsystem may be adapted from the vertical-plane subsystem previously presented by Tanakitkorn et al. (2016). The corresponding coefficients on the two subsystems are assumed to have the same magnitude, but they may feature different signs as to comply with the sign convention of the $b$-frame. These model parameters are detailed in Appendix B.

\subsection{Decoupled yaw dynamics}

The horizontal-plane dynamics may be simplified by assuming the decoupled yaw dynamics:

$$
\begin{aligned}
\left(I_{z z}-N_{\dot{r}}\right) \dot{r}-\left(N_{r}+N_{|r| r}|r|\right) r+\left(X_{\dot{u}}-Y_{\dot{v}}\right) u v-\left(N_{v}+N_{|v| v}|v|\right) v & =N, \\
\dot{\psi}-r & =0 .
\end{aligned}
$$

This may be rearranged into a conventional form of a state-space model as follows:

$$
\dot{\mathrm{x}}=\boldsymbol{A} \mathrm{x}+\boldsymbol{B} N+F(u, v),
$$

where state vector is denoted by $\mathrm{x}=[r, \psi]^{T}$ and coefficient matrices are given by:

$$
\begin{aligned}
\boldsymbol{A} & =\left[\begin{array}{cc}
I_{z z}-N_{\dot{r}} & 0 \\
0 & 1
\end{array}\right]^{-1}\left[\begin{array}{cc}
N_{r}+N_{|r| r}|r| & 0 \\
1 & 0
\end{array}\right], \\
\boldsymbol{B} & =\left[\begin{array}{cc}
I_{z z}-N_{\dot{r}} & 0 \\
0 & 1
\end{array}\right]^{-1}\left[\begin{array}{l}
1 \\
0
\end{array}\right] .
\end{aligned}
$$

The terms that do not involve either the state vector or the control moment are grouped together as,

$$
F(u, v)=\left[\begin{array}{cc}
I_{z z}-N_{\dot{r}} & 0 \\
0 & 1
\end{array}\right]^{-1}\left[\begin{array}{c}
-\left(X_{\dot{u}}-Y_{\dot{v}}\right) u v+\left(N_{v}+N_{|v| v}|v|\right) v \\
0
\end{array}\right] .
$$




\section{Sliding mode heading control design}

In this section, the control system is designed using the state-space representation of the decoupled yaw dynamics, equation (7). The design process can be broken down into three steps. Firstly, a sliding variable is chosen to represent the desired response. Secondly, a control law is devised, generating a generalised control moment that directs the system's state to reach and glide along the sliding surface. Thirdly, a control allocation is imposed to effectively share the generalised control moment between the thrusters and the rudders. A sliding mode heading control block diagram is illustrated in figure 3; each component is explained later in this section.

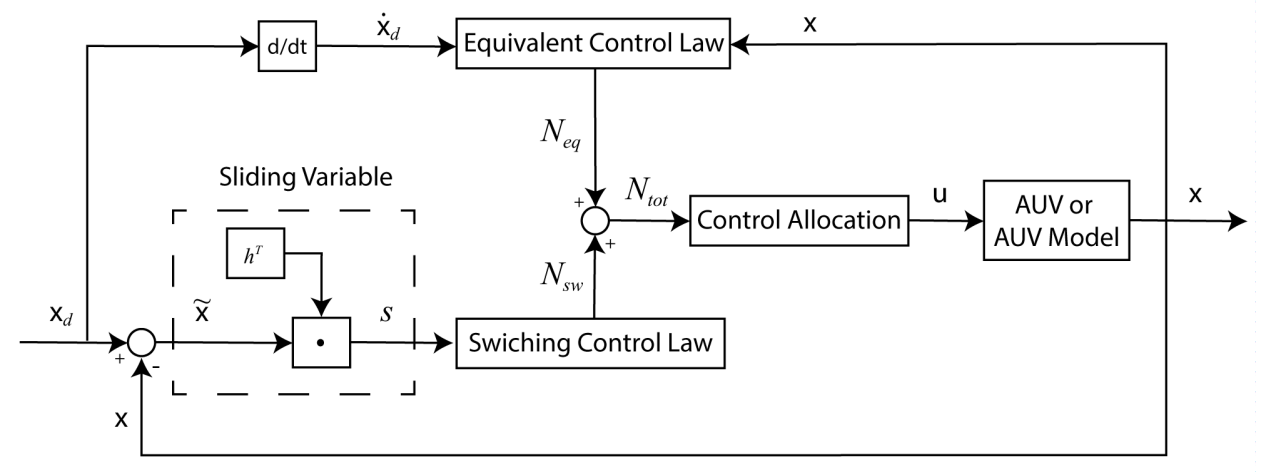

Figure 3: A sliding mode heading control block diagram.

\subsection{Sliding variable}

An expected behaviour of the system is when the heading is maintained steadily with no heading error. On this account, a desired state vector is, $\mathrm{x}_{d}=\left[r_{d}, \psi_{d}\right]^{T}$, and a state error vector is, $\tilde{\mathrm{x}}=\mathrm{x}-\mathrm{x}_{d}=[\tilde{r}, \tilde{\psi}]^{T}$. A sliding variable is then chosen to be (Yoerger and Slotine, 1985; DeCarlo et al., 1988; Fossen, 2011):

$$
s=h^{T} \tilde{\mathrm{x}}=\left[h_{1}, h_{2}\right]\left[\begin{array}{c}
\tilde{r} \\
\tilde{\psi}
\end{array}\right]=h_{1} \tilde{r}+h_{2} \tilde{\psi},
$$

and the sliding surface is where $s=0$. This form of the sliding variable could be considered to have a low-pass characteristic with a bandwidth of $h_{2} / h_{1}$ that defines how well the system could tolerate to high-frequency noises. A vector $h$ with a small bandwidth may be used to attenuate the oscillatory response (Shi et al., 2008).

\subsection{Control law}

A control law's role is to generate a generalised moment that directs the system to move toward and stay on the sliding surface, where $s=0$. It is designed to include two additive terms between equivalent control law and switching control law:

$$
N_{t o t}=N_{e q}+N_{s w}
$$

\subsubsection{Equivalent control law}

This equivalent control law, $N_{e q}$, generates a continuous control signal that directs the system towards the equilibrium, where $\dot{s}=0$ (Utkin et al., 2009; Spurgeon, 2014). 
A time derivative of the sliding variable, (11), is expressed:

$$
\dot{s}=h^{T} \dot{\mathrm{x}}-h^{T} \dot{\mathrm{x}}_{d} .
$$

By substituting (7) into (13), this yields:

$$
\dot{s}=h^{T}[\boldsymbol{A x}+\boldsymbol{B} N+F(u, v)]-h^{T} \dot{\mathrm{x}}_{d} .
$$

The equivalent control law is a solution to the above equation, where $\dot{s}=0$ :

$$
N_{e q}=\left(h^{T} \boldsymbol{B}\right)^{-1}\left[h^{T} \dot{\mathrm{x}}_{d}-h^{T} F(u, v)-h^{T} \boldsymbol{A x}\right] .
$$

\subsubsection{Switching control law (sign)}

The switching control law, $N_{s w}$, is imposed to provide a discontinuous control signal that changes between two states (turning left or right for instance) and forces the system to glide along the sliding surface. This term also provides robustness against the disturbances and parameter uncertainties. It is given by the form:

$$
N_{s w}=\left(h^{T} \boldsymbol{B}\right)^{-1}\left[K_{s w} \operatorname{sign}(s)\right]
$$

where $K_{s w}$ is the gain to modify the aggressiveness of the switching action. In the result section of this paper, this choice of switching function is referred to as "sign".

\subsection{Control allocation}

A circle manoeuvre was used for testing the AUV's yaw response when executing different combinations of propeller $\left(\mathrm{u}_{\text {prop }}\right)$, thruster $\left(\mathrm{u}_{t h}\right)$ and rudder $\left(\delta_{R}\right)$ setpoints. A steady turning rate and manoeuvring power (due to thrusters and rudders) corresponding to each combination were recorded and plotted against each other, see figure 4. The red lines in this figure indicate Pareto frontiers, which are a set of non-dominated options for the AUV to turn at a certain speed by spending least energy. (It is impossible to further improve any of these options to achieve either faster response or less control energy without sacrificing the other performance index.) These Pareto frontiers correspond to red dashed lines in figure 5. This suggests that it is more energy efficient to use the rudders as the main choice for heading control till they reach their effectiveness limit, and the thrusters that consume significantly more energy should be operated only when needed.

This control allocation strategy is implemented using daisy-chaining technique (Oppenheimer et al., 2006), where the diagram is illustrated in figure 6 . Given the generalised yaw moment, $N_{\text {tot }}$, from the control law, the rudder angle, $\delta_{R}$, is determined by using an inverse of the rudder model that is analogous to that explained by Tanakitkorn et al. (2016):

$$
\delta_{R}=\frac{N_{t o t}}{N_{|u| u \delta_{R}}|u| u} .
$$

When the rudder angle saturates, an actual moment generated by the rudders, $N_{R}$, is computed using the rudder model. A different yaw moment, $N_{d i f}=N_{t o t}-N_{R}$, is computed accordingly. This difference is further allocated onto the thrusters as follows:

$$
\mathrm{u}_{t h}=\operatorname{sign}\left(N_{d i f}\right) \sqrt{\frac{\left|N_{d i f}\right|}{L_{h} \rho D_{t h}^{4} K_{T, t h}}},
$$

where $L_{h}$ is a spacing between the two horizontal thrusters. Note that the above equation is derived from thruster model explained by Tanakitkorn et al. (2016), where deduction factors are omitted. 


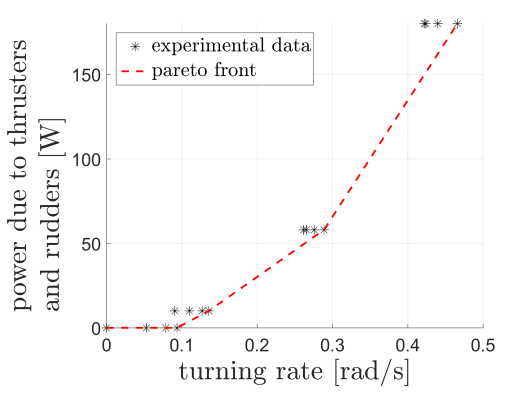

(a) $\mathrm{u}_{\text {prop }}=10$

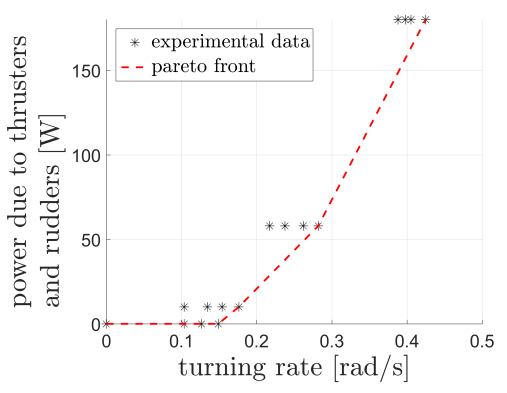

(b) $\mathrm{u}_{\text {prop }}=16$

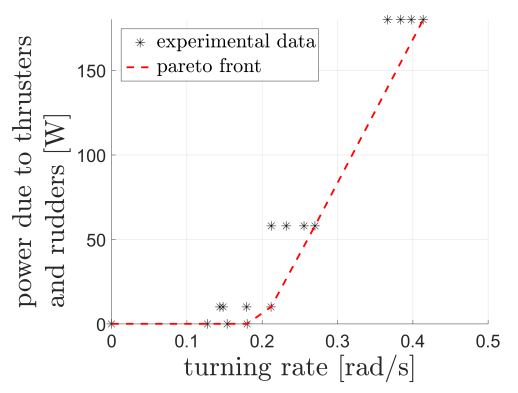

(c) $\mathrm{u}_{\text {prop }}=22$

Figure 4: Pareto frontier that highlights the optimal actuator setting to achieve a particular turning rate with a minimum manoeuvring power.

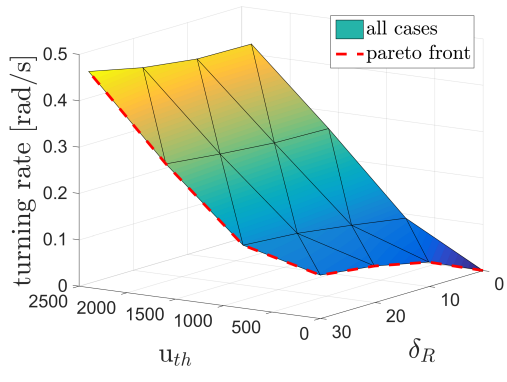

(a) $\mathrm{u}_{\text {prop }}=10$

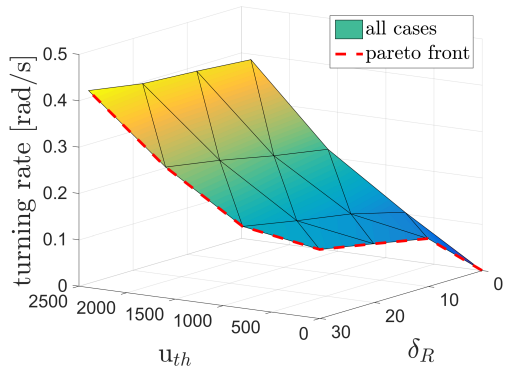

(b) $\mathrm{u}_{\text {prop }}=16$

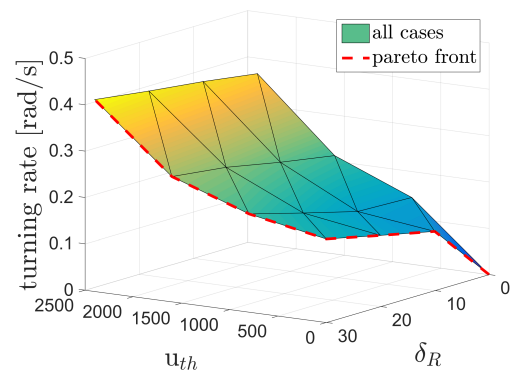

(c) $\mathrm{u}_{\text {prop }}=22$

Figure 5: Yaw response at speeds associated with Pareto frontier.

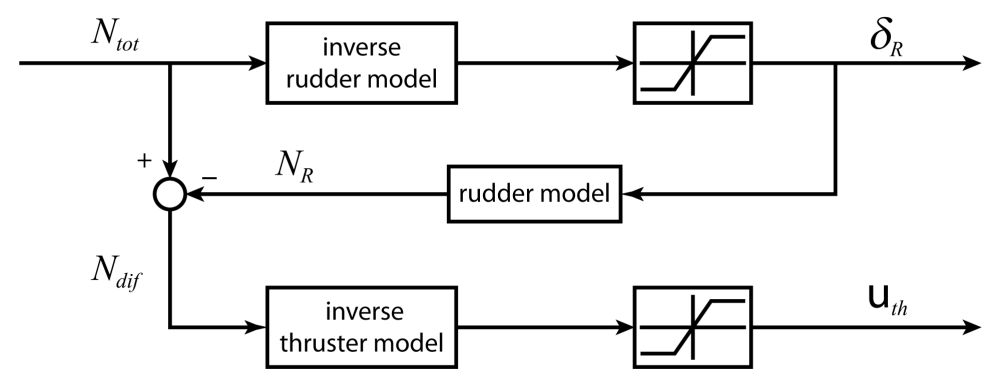

Figure 6: A daisy-chaining control allocation diagram.

\subsection{Stability analysis}

A Lyapunov stability criterion may be used for analysing the stability of the sliding mode control system. To this end, a following Lyapunov candidate function is chosen to represent both the turning velocity and the heading error:

$$
V=\frac{1}{2} s^{2}
$$

A time derivative of the Lyapunov function is

$$
\dot{V}=s \dot{s}
$$


By substitute the control law, (12), into a time derivative of the sliding variable, (14), this yields:

$$
\dot{s}=h^{T} \boldsymbol{A} \mathrm{x}+h^{T} \boldsymbol{B} N_{e q}+h^{T} \boldsymbol{B} N_{s w}+h^{T} F-h^{T} \dot{\mathrm{x}}_{d} .
$$

Further substitute the equivalent control law, (15), and the switching control law, (16), into the above equation. This gives:

$$
\begin{aligned}
\dot{s} & =h^{T} \boldsymbol{A} \mathrm{x}+h^{T} \dot{\mathrm{x}}_{d}-h^{T} F-h^{T} \boldsymbol{A} \mathrm{x}-K_{s w} \operatorname{sign}(s)+h^{T} F-h^{T} \dot{\mathrm{x}}_{d} \\
& =-K_{s w} \operatorname{sign}(s)+\epsilon,
\end{aligned}
$$

where most terms are cancelled out, and $\epsilon$ represents a mismatch between the estimated and the actual state of the system.

The system is said to be stable if $\dot{V}<0$. This condition guarantees that the dynamics and heading error are decaying whilst the system is driven toward the sliding surface and remain on the origin thereafter. Based on this condition, we have,

$$
\begin{aligned}
\dot{V} & =s \dot{s} \\
& =-s K_{s w} \operatorname{sign}(s)+s \epsilon<0 .
\end{aligned}
$$

This suggests that the system stability is guaranteed if,

$$
K_{s w}>|\epsilon| .
$$

In other words, the switching gain has to be sufficiently large to overcome the disturbances and model uncertainties.

\section{Chattering alleviation}

A typical switching control law involves a discontinuous control signal that likely causes the system to chatter (see figure 2), especially when the switching gain is large. This results in a high actuator wear and also an increase in energy consumption. In this section, three alternative approaches are highlighted for reducing the severity of the chattering.

\subsection{Continuous approximation (con)}

The chattering can be attenuated if the discrete switching function is made continuous over a small vicinity around the sliding surface. This may be done by replacing the signum function in the switching control law with a hyperbolic tangent function (Vuilmet, 2005; Jantapremjit and Wilson, 2008):

$$
N_{s w}=-\left(h^{T} \boldsymbol{B}\right)^{-1}\left[K_{s w} \tanh \left(\frac{s}{\sigma^{*}}\right)\right],
$$

where a boundary layer thickness, $\sigma^{*}$, is used to control a transition band, see figure 7 .

In the result section of this paper, this choice of switching function is referred to as "con".

\subsection{Super-twisting algorithm (STA)}

The super-twisting algorithm may be categorised as a second-order sliding mode control that helps to restrain the chattering effect while maintaining a high robustness. However, unlike other higher-order sliding mode 


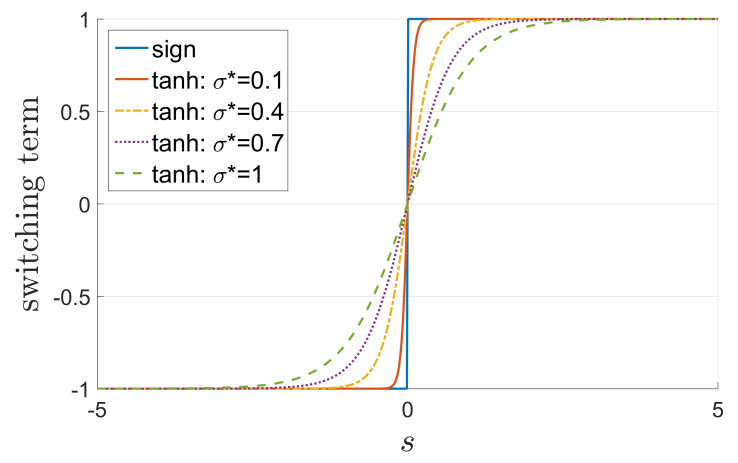

Figure 7: An influence of a boundary layer on the transition band.

approaches, it does not require a higher-order derivative of the sliding variable; hence, it is less sensitive to system noises. The algorithm takes the form (Levant et al., 2000; Shtessel et al., 2014; Han and Liu, 2016):

$$
N_{s w}=-\left(h^{T} \boldsymbol{B}\right)^{-1}\left[K_{s w, 1} s^{1 / 2} \operatorname{sign}(s)+\int K_{s w, 2} \operatorname{sign}(s) \mathrm{d} t\right] .
$$

This STA switching control law consists of two terms (see figure 8): the first term features a time-varying gain that changes with $s^{1 / 2}$, producing a more gentle control demand when getting close to a sliding surface; the second term features an integrator that provides a continuous control signal and eliminates steady-state error.

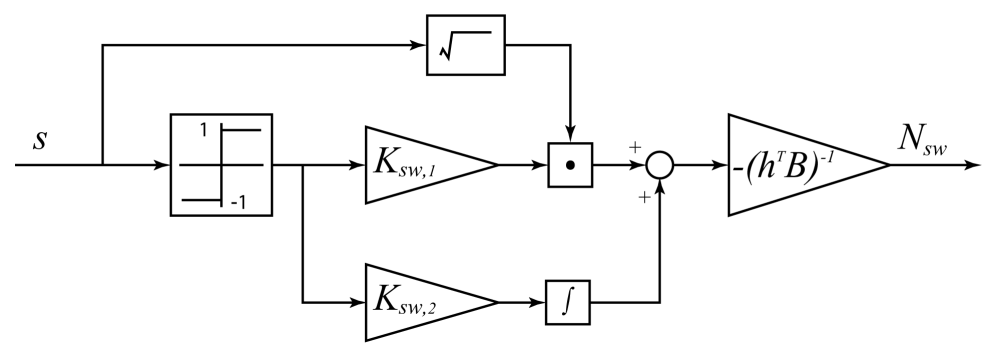

Figure 8: A super-twisting algorithm control block diagram.

\subsection{Simplified super-twisting algorithm (sSTA)}

In this approach, the STA approach is combined with a continuous approximation approach. Signum functions in (26) are altered by a hyperbolic tangent function with the boundary layer. Also, the time-varying gain is removed from the first term of the STA approach since hyperbolic tangent function alone can produce a more gently control action when getting close to the sliding surface. (See control block diagram in figure 9.) The sSTA switching control law takes the form:

$$
N_{s w}=-\left(h^{T} \boldsymbol{B}\right)^{-1}\left[K_{s w, 1} \tanh \left(\frac{s}{\sigma^{*}}\right)+\int K_{s w, 2} \tanh \left(\frac{s}{\sigma^{*}}\right) \mathrm{d} t\right] .
$$

\section{Simulation studies}

Influences of different switching functions and parameter uncertainties on control performance were studied. This was performed using Matlab based on the horizontal-plane dynamics model described in section 2, and 


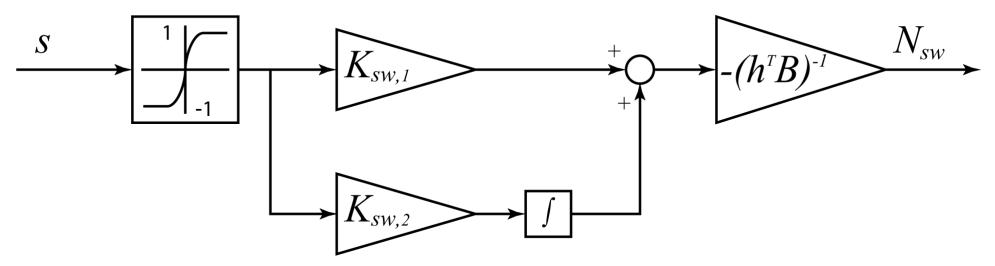

Figure 9: A simplified super-twisting algorithm control block diagram.

with the parameters provided in appendix B. The solver was Runge-Kutta $4^{\text {th }}$ order. Sample rates were fixed by IMU at $20 \mathrm{~Hz}$ for the dynamic modelling section and $5 \mathrm{~Hz}$ for the control section. These comply with actual sample rates used on the Delphin2 AUV. Initial conditions were $\eta=[0,0,0]^{T}$, i.e., the AUV was at rest. In each simulation case, the AUV underwent consecutive heading demand angles of 90, 120, 90, 180 and $90 \mathrm{deg}$ at an interval of $40 \mathrm{sec}$. These correspond to heading change of \pm 30 and $\pm 90 \mathrm{deg}$, respectively.

\subsection{Choice of switching function}

The four candidate switching functions are considered: sign, con, STA and sSTA. (They are described in section 3.2.2 and 4.) The controller gains used during the simulation are given in appendix D. They were chosen by a process of trial and error. Two testing scenarios are presented below to examine the influence of these switching functions on the sliding mode control performance.

\subsubsection{Without external disturbances}

The first scenario was operation at zero speed, where disturbances were neglected. Transient response for each approach is presented in figure 10 and steady-state performances is given in table 1. All four switching functions yielded similar rise times (less than $6 \mathrm{sec}$ for 90 deg heading change) with no overshoot. However, the signum approach caused the system to chatter very badly: the actual heading constantly fluctuated within a band of $2.5 \mathrm{deg}$ around the desired heading. This fluctuation was improved when the STA approach was used, yielding smaller oscillation bands in both the AUV dynamics and the control moment. In contrast, the fluctuation was totally eliminated when the continuous approximation or sSTA approach was used.

Figure 10c compares system state's trajectories for difference approaches. Signum and continuous approximation approaches took similar trajectories back to the origin. However, the signum approach featured a chaotic trajectory around the origin, which represented a chattering phenomenon, whereas continuous approximation approach nicely converged to the sliding surface and stayed at the origin thereafter. On the other hand, STA and sSTA took similar trajectories that went beyond the sliding surface, and then suddenly twisted back to the surface when getting close to the origin; this corresponds to a slightly smaller rise time as the AUV turned a little bit faster, and the turning velocity suddenly dropped when the desired heading was almost reached. 

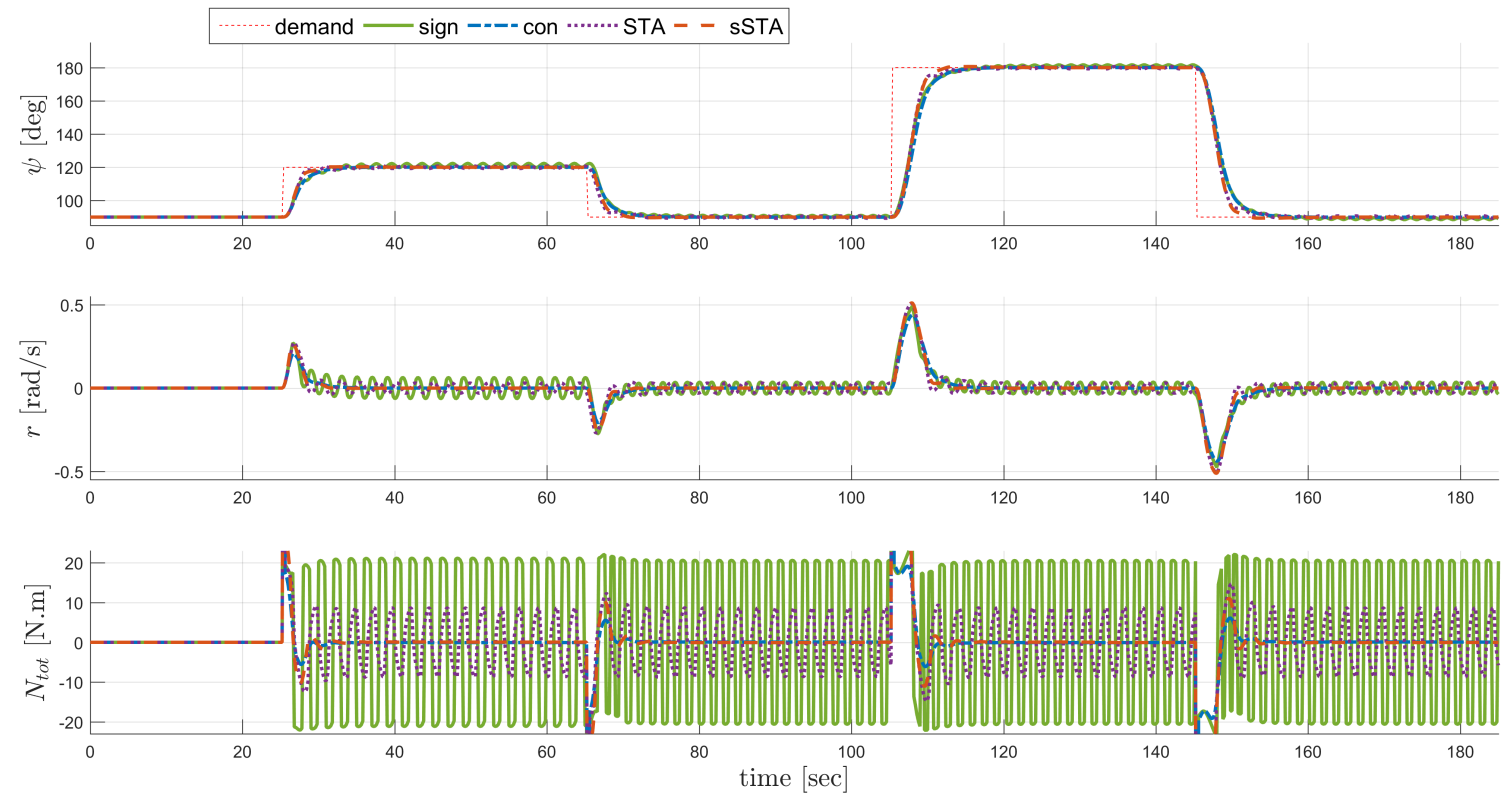

(a) time response
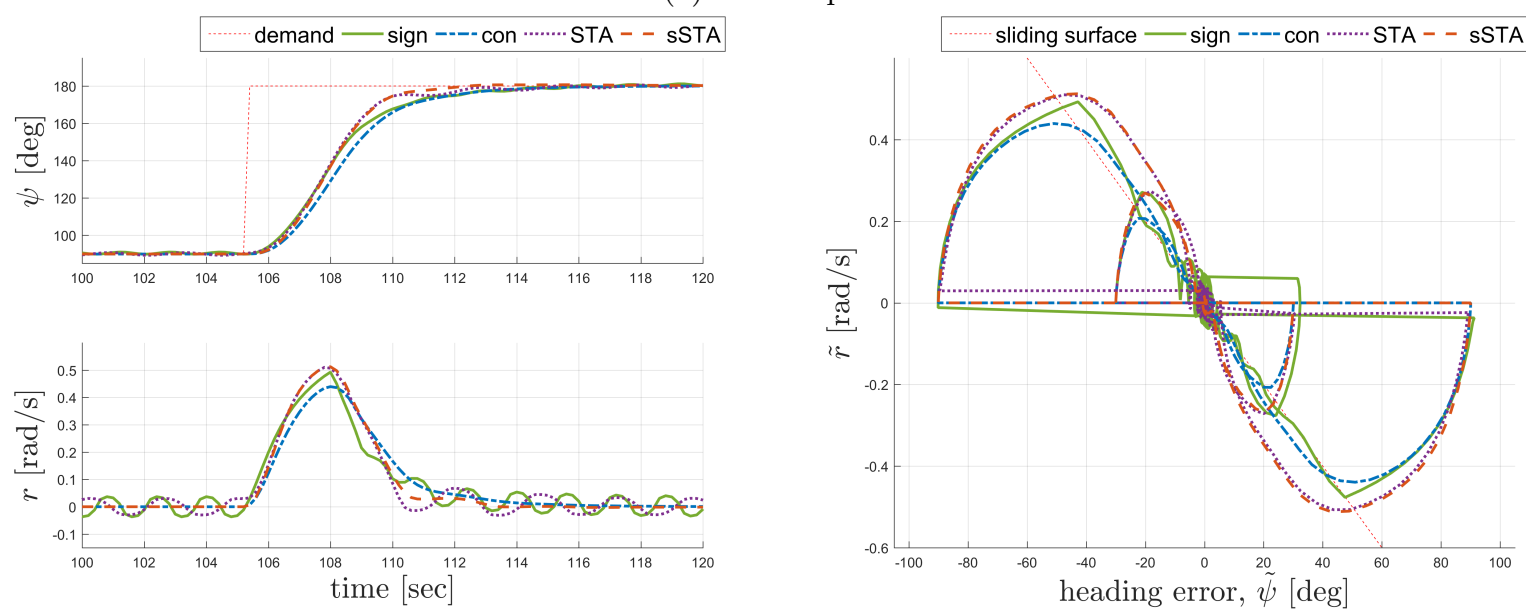

(b) time response (from $100^{\text {th }}$ to $120^{\text {th }}$ second)

(c) phase plot

Figure 10: Simulated results at zero speed operation (external disturbances are neglected).

Table 1: Influences of switching functions on steady-state performance (external disturbances are neglected).

\begin{tabular}{|c|c|c|c|c|}
\hline \multirow{2}{*}{$\begin{array}{l}\text { switching } \\
\text { function }\end{array}$} & \multicolumn{3}{|c|}{ band of variation } & \multirow{2}{*}{$\begin{array}{c}\tilde{\psi} \\
{[\mathrm{deg}]}\end{array}$} \\
\hline & $\psi[\mathrm{deg}]$ & $r[\mathrm{rad} / \mathrm{s}]$ & $N[\mathrm{~N} \cdot \mathrm{m}]$ & \\
\hline sign & 2.5 & 0.07 & 42.41 & 1 \\
\hline con & 0 & 0 & 0 & 0 \\
\hline STA & 1.4 & 0.06 & 17.25 & 0 \\
\hline SSTA & 0 & 0 & 0 & 0 \\
\hline
\end{tabular}




\subsubsection{With a constant external disturbance}

This scenario simulates AUV operation at zero speed with constant external disturbances of $10 \mathrm{~N} \cdot \mathrm{m}$ applied. This aims to investigate the influence of the switching functions when the system is exposed to the environmental disturbance. Transient response for each approach is presented in figure 11 and steady-state performances is given in table 2 .
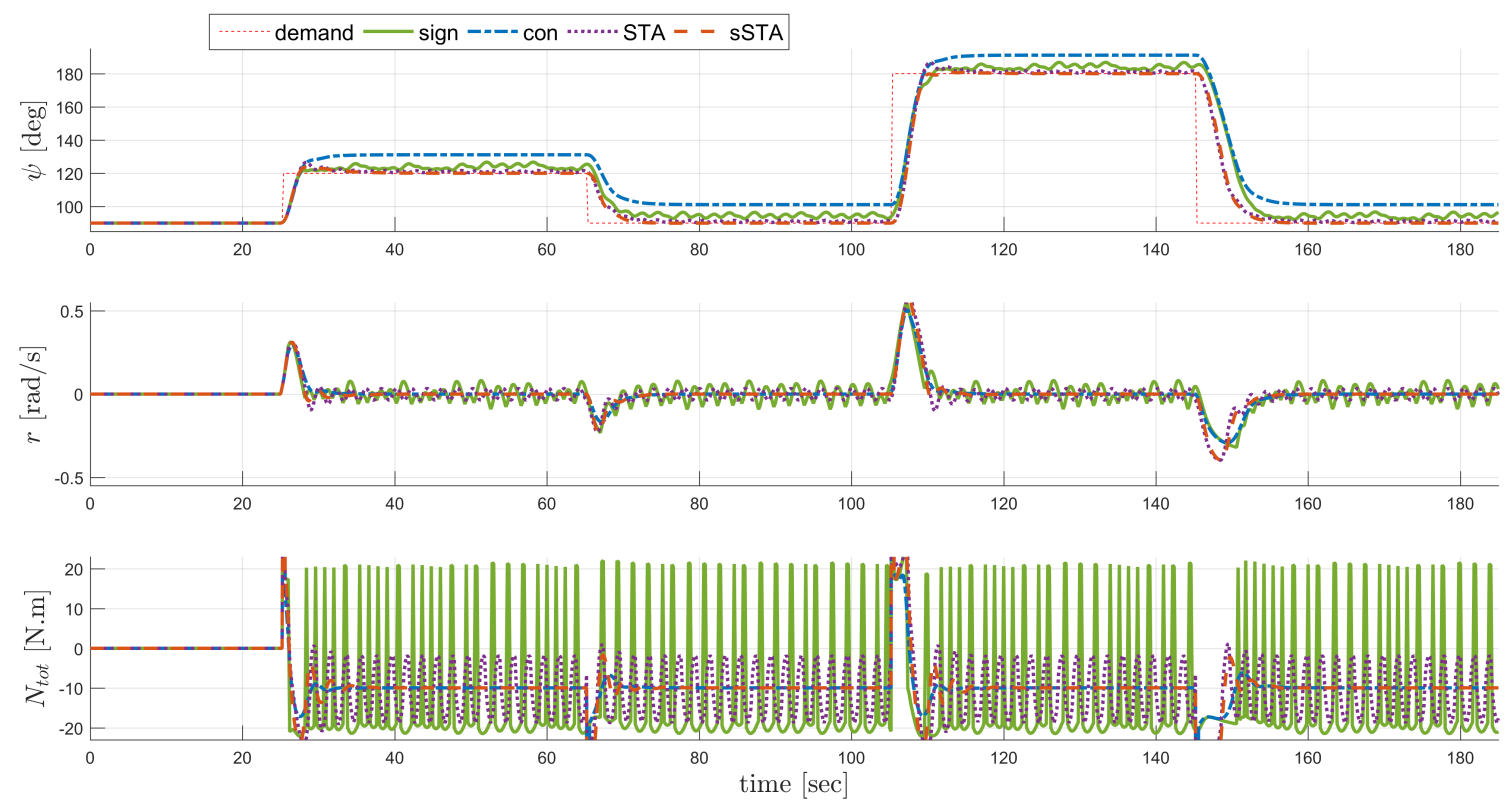

(a) time response
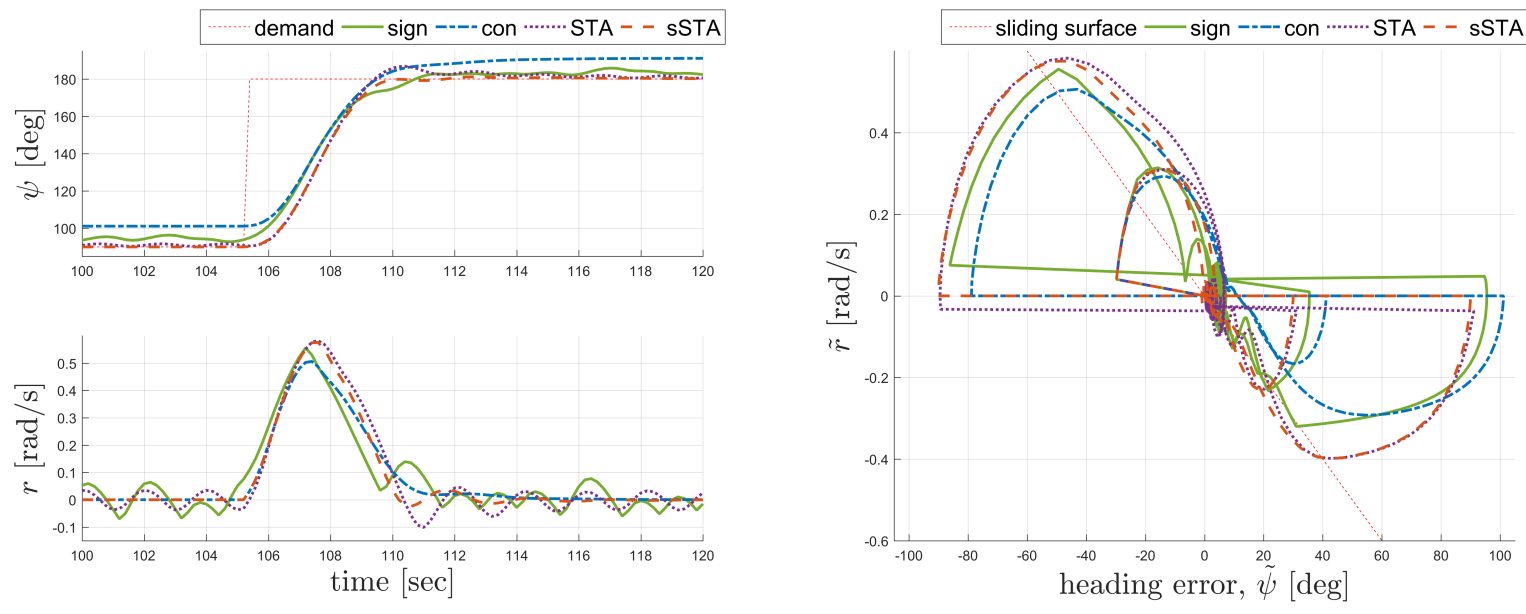

(b) time response (from $100^{\text {th }}$ to $120^{\text {th }}$ second)

(c) phase plot

Figure 11: Simulated results at zero speed operation $(10 \mathrm{~N} \cdot \mathrm{m}$ yaw disturbance is applied).

According to figure 11a, signum and continuous approximation approaches exhibited a constant fluctuation in the control moment with a fluctuation band of 42.43 and $16.97 \mathrm{~N} \cdot \mathrm{m}$ respectively, and with a bias towards negative value against the external disturbance. They also yielded a steady-state heading error of 4.8 and 11.1 deg respectively. In contrast, STA and sSTA generated precisely $-10 \mathrm{~N} \cdot \mathrm{m}$ control moment that cancelled out the external disturbance; hence the system exhibited no steady-state error. Considering the chattering problem, the signum approach produced a high-frequency oscillation in the AUV dynamics. This oscillation 
Table 2: Influences of switching functions on steady-state performance (10N·m yaw disturbance is applied).

\begin{tabular}{|l|r|r|r|r|}
\hline \multirow{2}{*}{$\begin{array}{l}\text { switching } \\
\text { function }\end{array}$} & \multicolumn{3}{|c|}{ band of variation } & \multirow{\psi}{*}{$\begin{array}{r}\psi \\
\text { [deg] }\end{array}$} \\
\cline { 2 - 5 } & $\psi[\mathrm{deg}]$ & $r[\mathrm{rad} / \mathrm{s}]$ & $N[\mathrm{~N} \cdot \mathrm{m}]$ & 4.8 \\
\hline \hline sign & 3.76 & 0.17 & 42.41 & 4.8 \\
\hline con & 0 & 0.00 & 0.00 & 11.1 \\
\hline STA & 1.3 & 0.07 & 16.97 & 1.0 \\
\hline sSTA & 0 & 0.00 & 0.00 & 0.0 \\
\hline
\end{tabular}

was improved slightly when the STA approach was used; however, the fluctuation in both AUV dynamics and actuator demand are still considered unacceptably large. The continuous approximation and sSTA, on the other hand, yield absolutely no oscillation at all in the AUV dynamics and control demand.

Phase plot in figure 11c yields the same conclusions. The signum and continuous approximation approach offset from the origin, indicating a steady-state error. STA approach converged to and stayed over the origin with a chaotic trajectory over it; this indicates the chattering phenomenon. The sSTA approach gently directed the system state towards the origin with a negligible amount of oscillation, implying a good control performance and robustness against external disturbance.

\subsection{Sensitivity to parameter uncertainties}

The simulation results from the first two scenarios have suggested that the sSTA approach is the best choice among the candidates; it produced neither steady-state error, overshoot, nor oscillation even in the present of an external disturbance. Due to this, further investigation was made only for the sSTA approach. To investigate the effect of parameter uncertainties on the sliding mode control performance, eight key model parameters used in the control section were individually varied between $\pm 90 \%$ of the estimated values used in the simulation section. Only the results for sSTA switching function are presented since it has shown superior performance over other switching functions, see section 5.1. In each simulation case, the AUV was demanded to track the four consecutive heading changes ( \pm 30 and $\pm 90 \mathrm{deg}$ ) at high speed (approximately $1 \mathrm{~m} / \mathrm{s}$ ), where surge, sway and yaw dynamics are highly coupled. This corresponds to a constant propeller demand of $\mathrm{u}_{\text {prop }}=22$.

The results for $I_{z z}$ and $N_{|r| r}$ are presented in figures 12 and 13 - the other six parameters provide similar plots but their influence is not as much. In every simulation case, the system successfully converged to the desired heading within a finite time, yielding overall good performance even though the model parameters were seriously over/underestimated; however, the transient response did vary with the variation of the parameters used in the control section.

The moment of inertia, $I_{z z}$, had a prominent effect on the transient response, see figure 12a. Especially, when it was underestimated by $90 \%$, the system overshot by approximately $22 \%$, and the oscillation took about 8 sec longer to decay. When $I_{z z}$ was underestimated by $45 \%$, the heading overshoot reduced significantly to $4 \%$ with no oscillation observed. In contrast, overestimating in $I_{z z}$ yielded a positive effect on the control system because the transient did not vary much and remained acceptable even for $I_{z z}+90 \%$. The added moment of inertia, $N_{\dot{r}}$, also influenced the system in the same way as $I_{z z}$, but not as strongly because of its relatively smaller magnitude. The influence of two added masses, $X_{\dot{u}}$ and $Y_{\dot{v}}$, were negligibly small because they are not directly related to the yaw dynamics.

Considering the damping terms, the influence of $N_{v}$ and $N_{|v| v}$ on the transient was very subtle because these are cross-coupling terms. On the other hand, variation in $N_{r}$ and $N_{|r| r}$ highly influenced the transient. The case with $N_{r}$ overestimated by $90 \%$ yielded a maximum heading overshoot of $5 \%$. Similarly, $15 \%$ heading overshoot was observed when $N_{|r| r}$ was overestimated by $90 \%$. This trend is intuitive because the control system would overreact when the damping terms are overestimated. The opposite trend was observed 
when the damping terms were underestimated: the control system would react more gently, taking up to approximately $2 \mathrm{sec}$ longer to rise, but with a negligibly small heading overshoot.

Overall control moment for each case was quantified by integrating the generalised moment over time; they are compared in figure 14. There was a $40 \%$ increase in the control moment required to suppress the oscillation when $I_{z z}$ was underestimated by $90 \%$. There was also a significant variation in the control moment (between $-13 \%$ to $27 \%$ ) that caused when $N_{r}$ and $N_{|r| r}$ are wrongly estimated. Effects of other parameters on the overall control moment were considered to be unimportant.
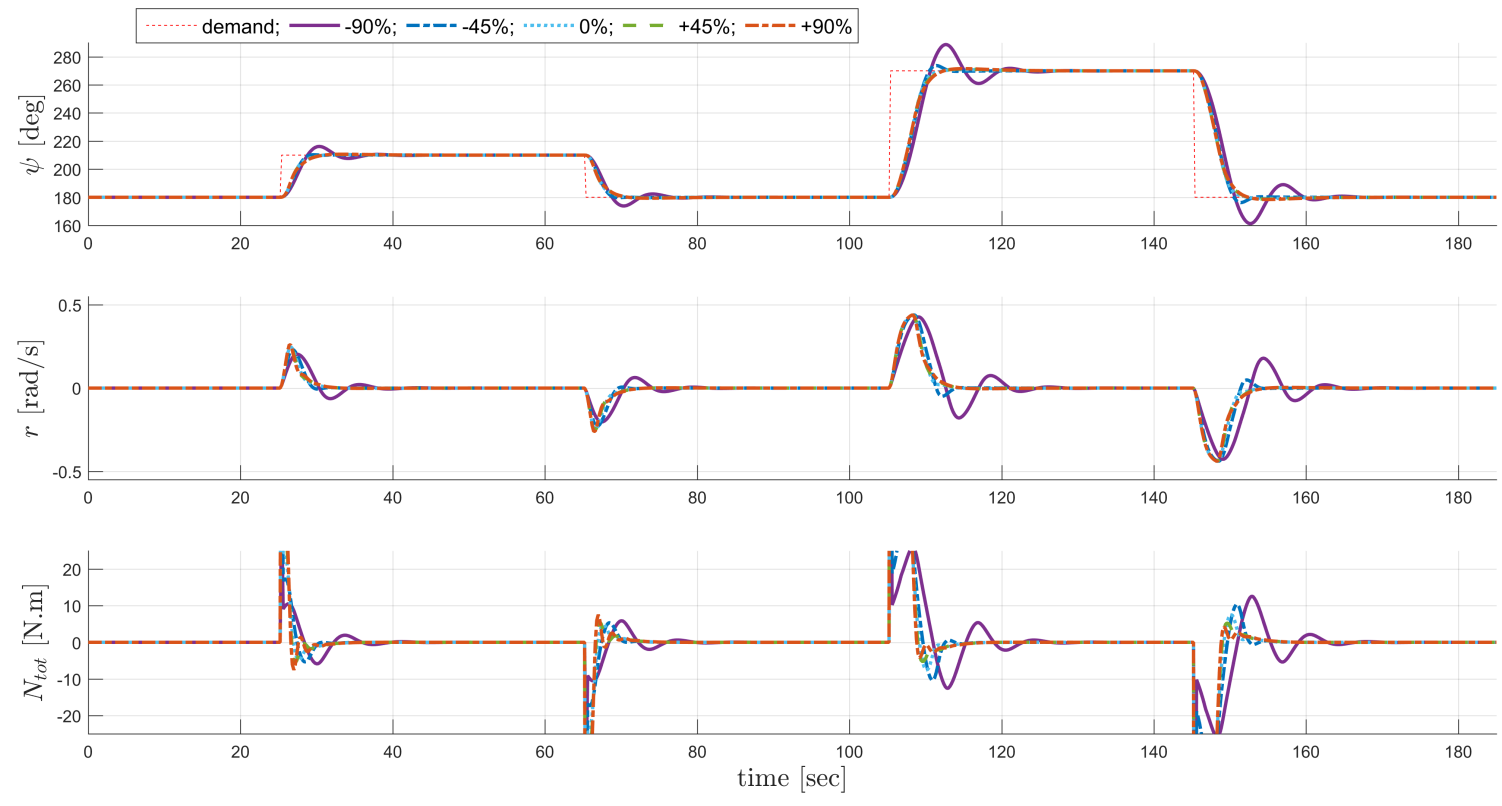

(a) time response
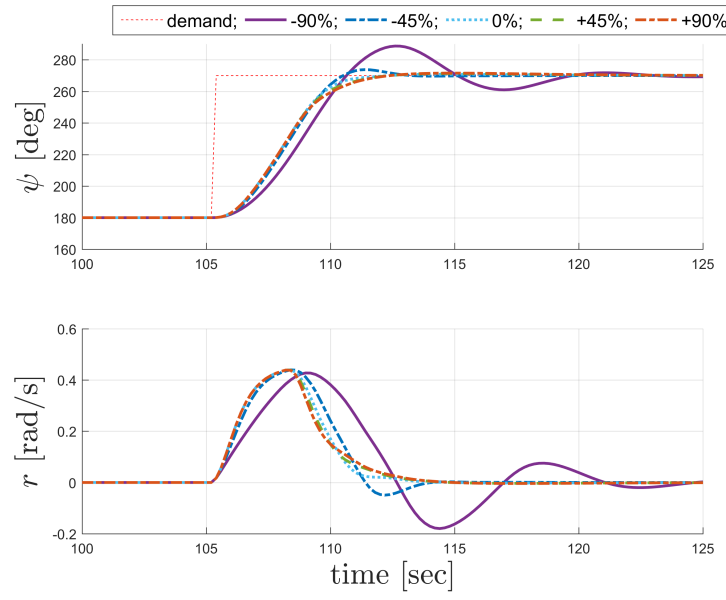

(b) time response (from $100^{\text {th }}$ to $125^{\text {th }}$ second)

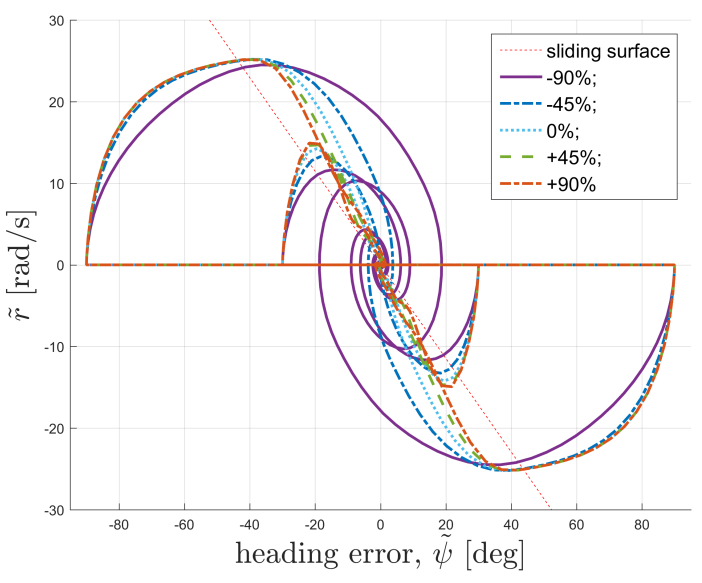

(c) phase plot

Figure 12: Influence of a variation in $I_{z z}$ on the control system. 

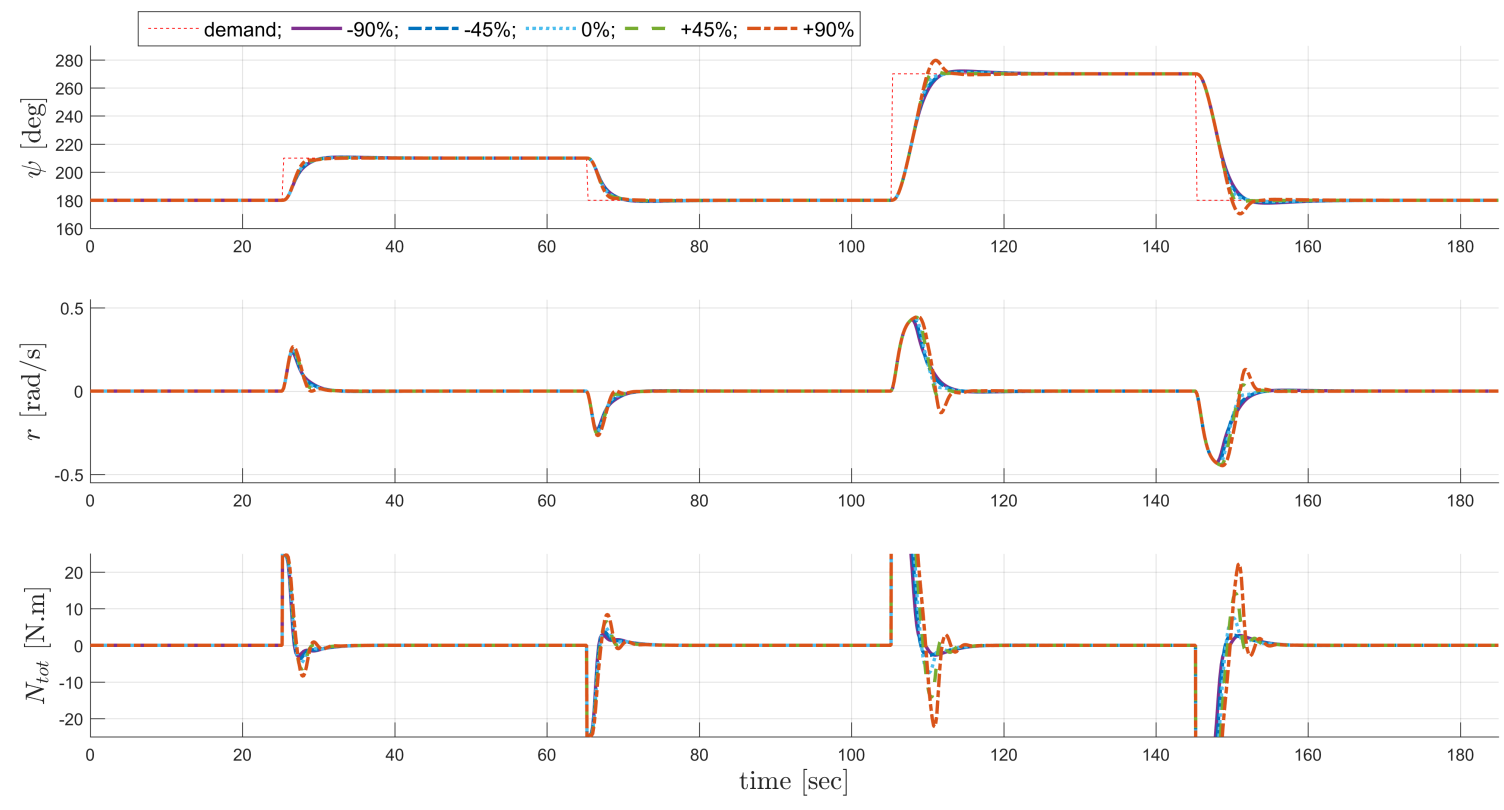

(a) time response

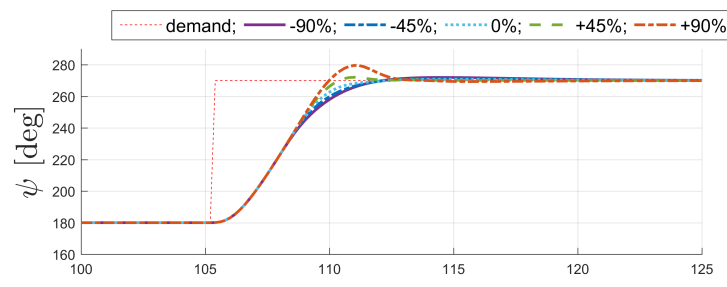

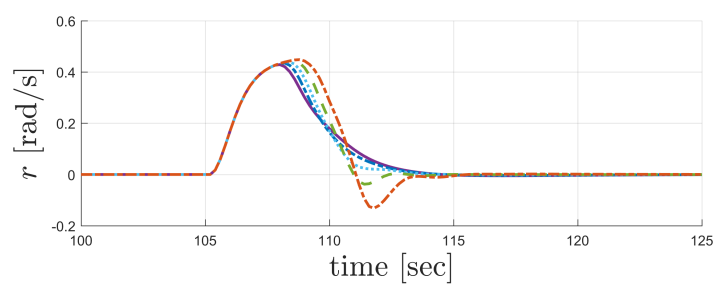

(b) time response (from $100^{\text {th }}$ to $125^{\text {th }}$ second)

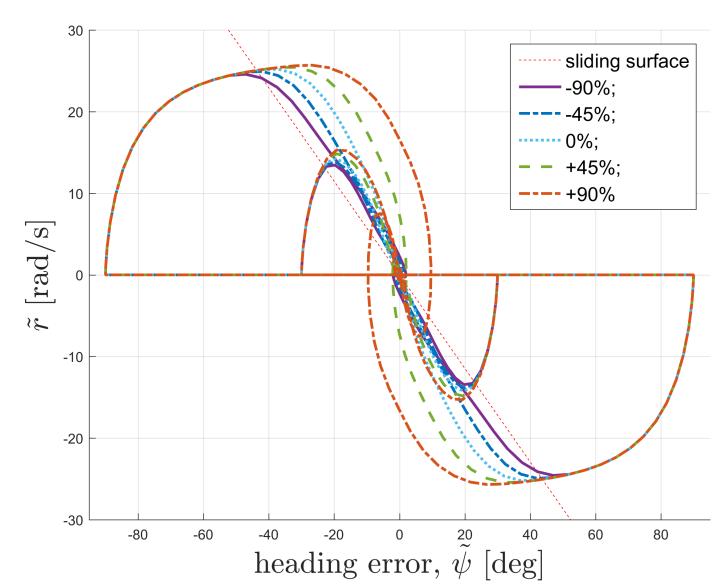

(c) phase plot

Figure 13: Influence of a variation in $N_{|r| r}$ on the control system.

\section{$6 \quad$ Experiment setup}

The proposed sliding mode heading control with the sSTA approach was verified on the Delphin2 AUV. The experiment was carried out in the freshwater man-made Eastleigh lake, Southampton, UK. A 90 deg sudden turn was used for evaluating the heading tracking performance. The tests were performed at a range of propeller setpoints, $\mathrm{u}_{\text {prop }}=\{0,10,16,22\}$, which correspond to forward speeds of $u=\{0,0.42,0.82,1.03\}$ $\mathrm{m} / \mathrm{s}$; they are referred to in the following section as zero-, low-, mid- and high-speed, respectively. The controller gains used during the simulation are given in appendix D. Power consumption for each run was recorded using a custom-built energy monitoring board that constantly measures voltages, and currents supplied to each set of actuators. Path following missions were proposed, aiming to demonstrate the control performance in a practical operation, where the heading control module collaborates with a guidance module. 


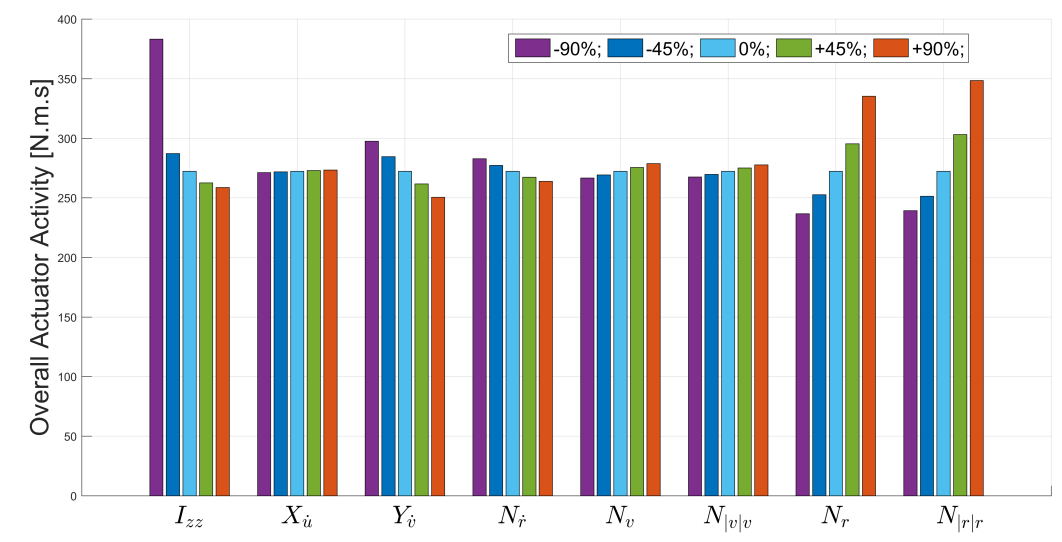

Figure 14: A variation in overall control moment due to parameter uncertainties.

The experiment was performed on the water surface, where a current position can be obtained from the GPS at a sample rate of $1 \mathrm{~Hz}$. A current heading and turning rate were obtained from the Xsens $4^{\text {th }}$ gen MTi-30 IMU at a sample rate of $20 \mathrm{~Hz}$. Forward and sway velocities were estimated using the 3DOF dynamic model described in section 2 .

During the experiment, Delphin2 was ballasted to be slightly positively buoyant by $+6 \mathrm{~N}$. This allows the vehicle just to float (submerge $99.24 \%$ by volume) and naturally return to the water surface to get the GPS fix.

\section{$7 \quad$ Results and discussions}

\subsection{Sudden turn}

In each run, the AUV was programmed to execute a constant propeller demand, tracking a constant heading setpoint for $20 \mathrm{sec}$. Then, the heading setpoint suddenly changed by $90 \mathrm{deg}$, and the AUV was now tracking this new heading setpoint while continuing to execute the same propeller demand for another 70 sec. Both simulation and experimental results for the proposed SMC scheme are presented and compared in figures 15 and 16. They are benchmarked against the experimental results obtained by using the P-D control law which was tuned experimentally, as described in appendix A, with the same control allocation technique.

In the experiment, the SMC scheme exhibited a slight variation in the actual heading that was confined within $\pm 2 \mathrm{deg}$, which is considered acceptable operationally. In the simulation, the heading remains absolutely stable. This difference was due to the measurement noise and environmental disturbance that the system was exposed to in the experiment. Otherwise, the experimental results from the SMC scheme agree very well to the simulation in that, for all cases, the system took 6 sec to rise and successfully converged to the desired heading with a negligibly small overshoot and with no steady-state error. Besides, in the phase plot, they produced similar trajectories that went beyond the sliding surface with a little twist back to the equilibrium when getting close to the origin.

In the low-speed cases, for both simulation and experiment, the generalised yaw moment was allocated to only the horizontal thrusters since the rudders were not effective in this regime. In mid- and high-speed cases, thrusters were operated only during a sudden turn as to fulfil a large control moment; otherwise, the control demand was allocated onto the rudders that consume significantly less power but work effectively and efficiently for the heading tracking. 
For the P-D scheme, it yielded satisfactory experimental results for the zero-speed case, which is where the controller is tuned. The system took $6.4 \mathrm{sec}$ to rise and could steadily maintain the desired heading with a negligibly small overshoot and no steady-state error. In this zero-speed case, it even outperformed the SMC scheme during the steady state: the generalised moment was more stable and the heading error was bound within $\pm 1 \mathrm{deg}$ as opposed to $\pm 2 \mathrm{deg}$ when the SMC was used. However, the P-D approach took 1 sec longer to rise. More importantly, the P-D control performance significantly degraded as forward speed increased. This become obvious especially in the high-speed case: the rudder demand repeatedly swung between \pm 20 deg in amplitude, which corresponded to the oscillation in the AUV heading between \pm 8 deg. (See figure 16a.) This is confirmed in the phase plot (figure 16c) that the P-D scheme yields a circular trajectory around the origin rather than staying on it. This circular trajectory implies a consistent oscillation during the steady state. Also, the centre of the circular trajectory shifted to the left by $3 \mathrm{deg}$, indicating the steady-state error, averagely. Gain schedule techniques may be used to extend an adequate control authority toward the highspeed regime. For example, the derivative gain may be set to increase with the forward speed in order to dampen the oscillatory response.

When comparing the P-D scheme to the presented SMC scheme, the differences in performance for zero- and low-speed cases are not obvious; however, the SMC performance is more consistent and does not degrade as the speed increases. This suggests robustness of the SMC scheme over the environmental disturbances and parameter uncertainties. Also, the proposed SMC scheme does not exhibit strong jerky motion, unlike the conventional SMC variants in the literature. Such a smooth control response highly benefits AUV operation, e.g., to obtain a good quality seafloor map with a sidescan sonar.

The damping coefficients and inertia terms for fully-submerged operation are expected to be different than that on the water surface. This will affect the control performance. However, the evidence provided in section 5.2 suggests that the SMC scheme can tolerate to an extreme level of parameter uncertainties and it is capable of maintaining the robust control performance over the possible range of the parameter values. 

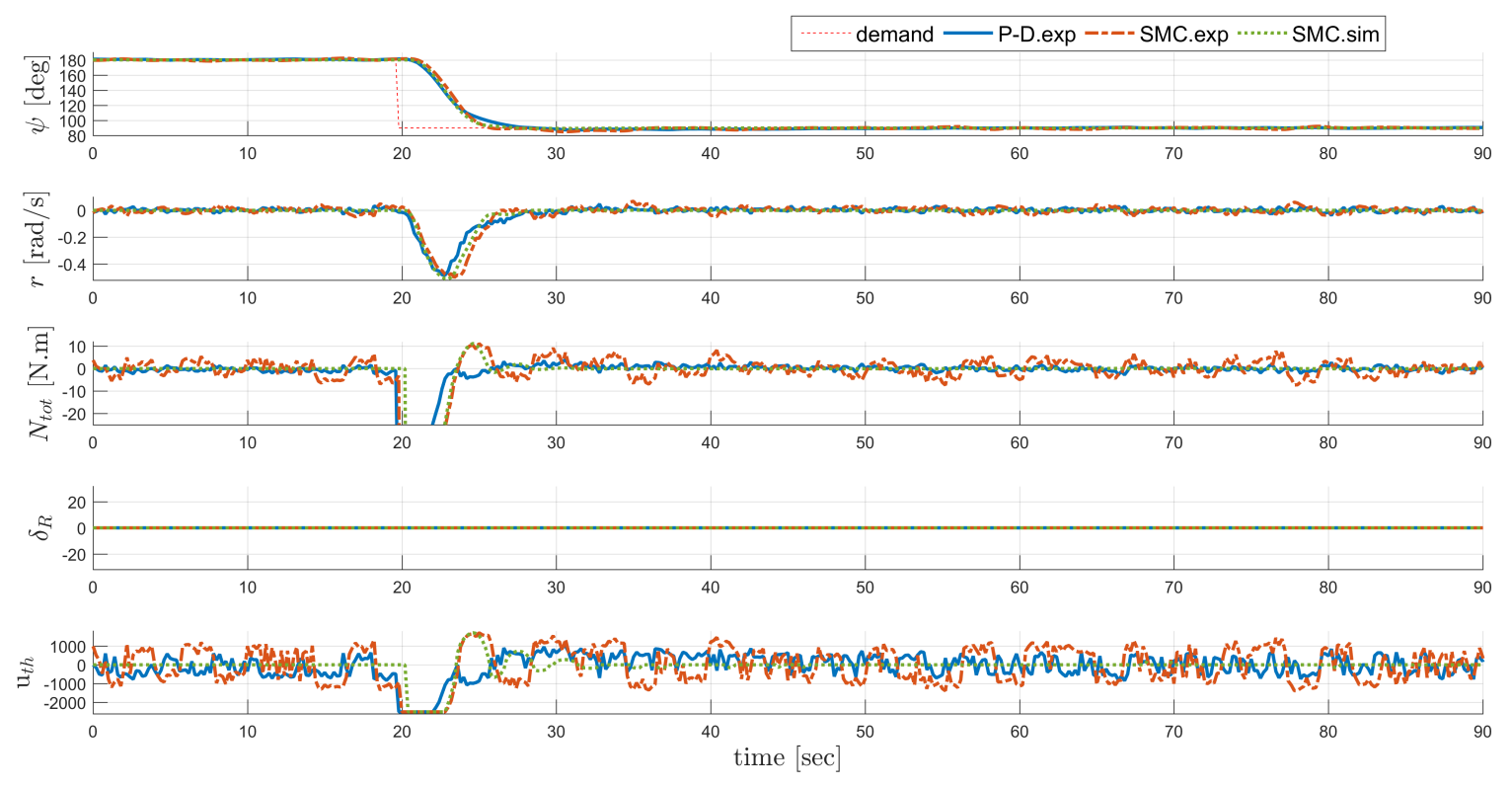

(a) time response
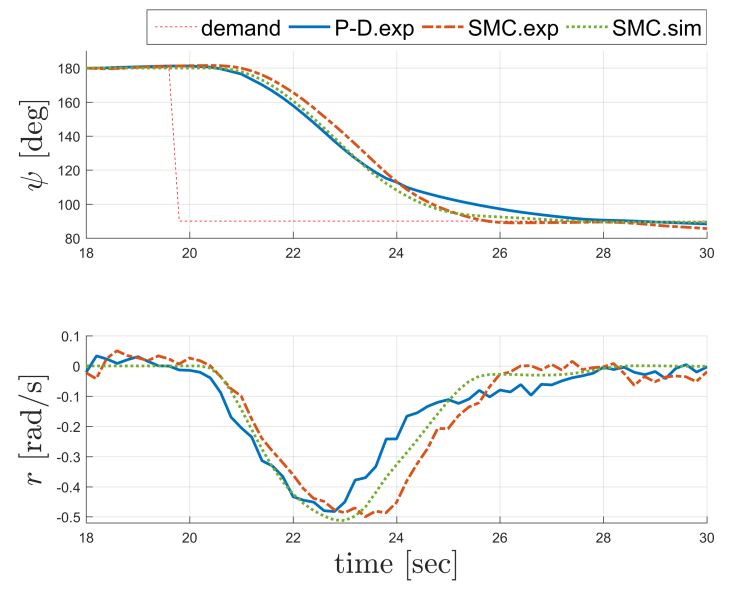

(b) time response (from $18^{\text {th }}$ to $30^{\text {th }}$ second)

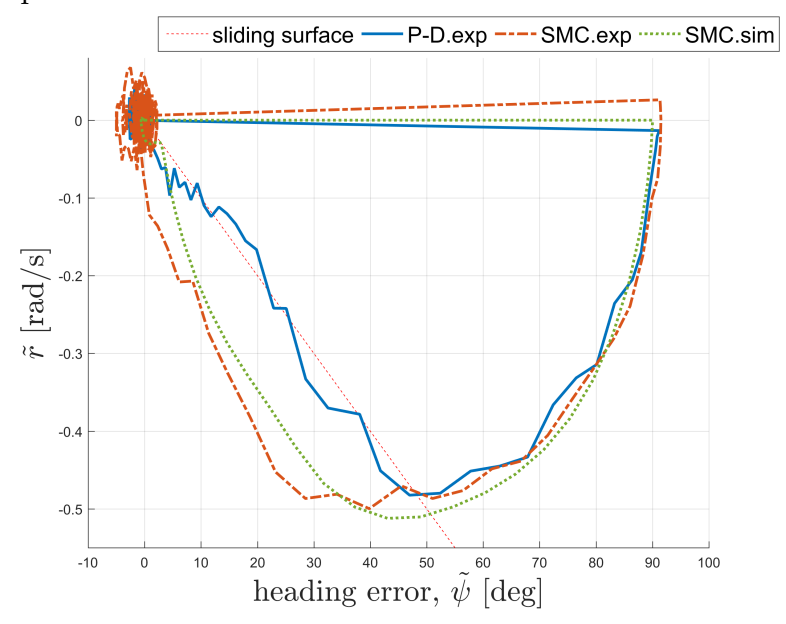

(c) phase plot

Figure 15: Comparison on sudden turn results $\left(\mathrm{u}_{\text {prop }}=0\right)$; both approaches provided similar performance in that they took less than $8 \mathrm{sec}$ to rise and the heading was maintained steadily thereafter. 

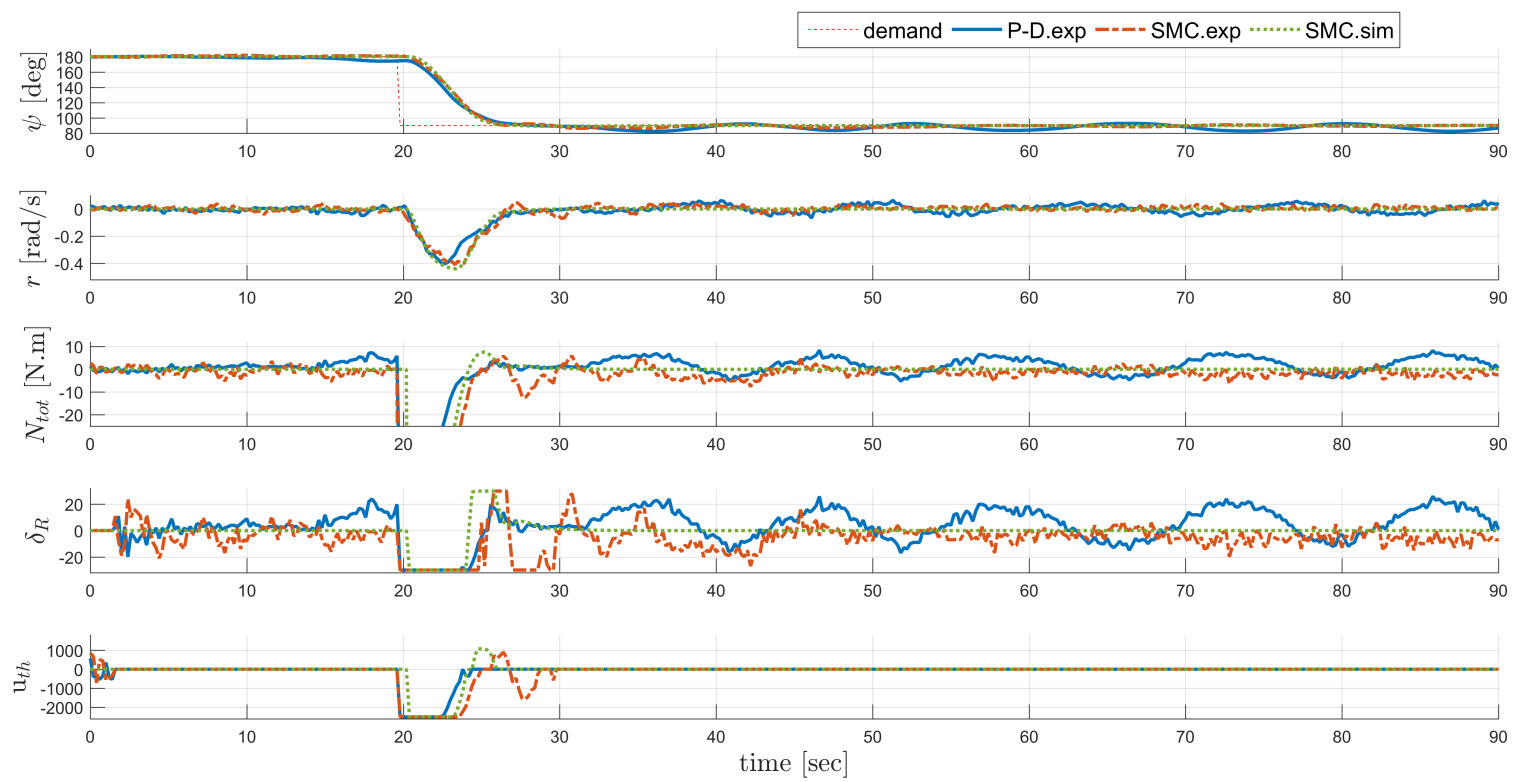

(a) time response
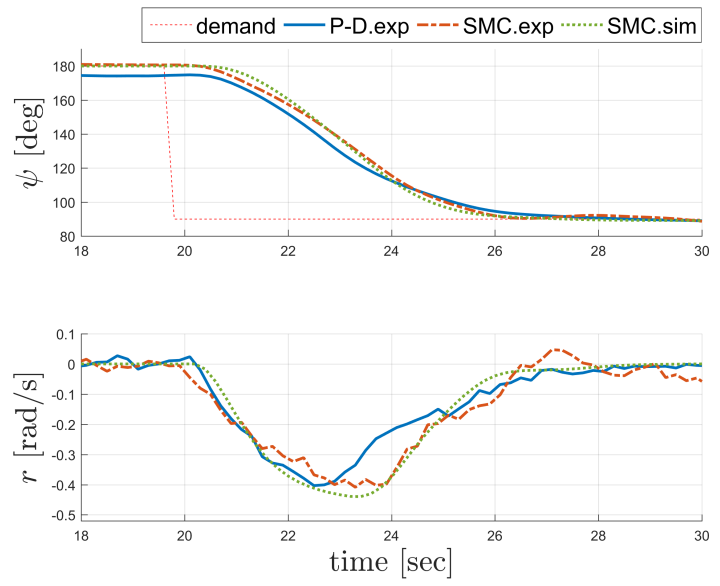

(b) time response (from $18^{\text {th }}$ to $30^{\text {th }}$ second)

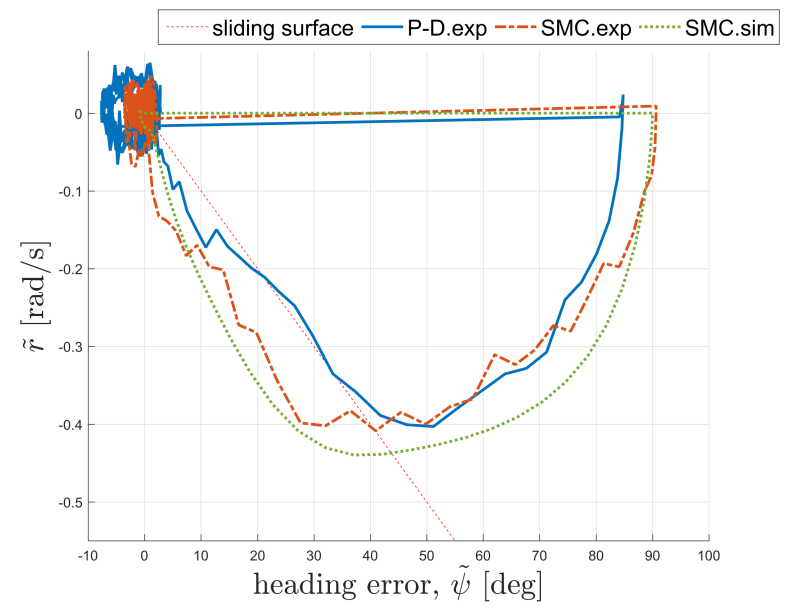

(c) phase plot

Figure 16: Comparison on sudden turn results $\left(\mathrm{u}_{\text {prop }}=22\right)$; the P-D yielded an oscillatory response whereas the SMC provided roughly the same performance as that of the zero-speed case. 


\subsection{Power consumption for the sudden turn tests}

Power consumption profiles corresponding to the experimental results for SMC and P-D schemes in the previous subsection are presented in figure 17. Manoeuvring power, $P_{M}$, is used to denote the total power required for steering the AUV: this includes power spent on the horizontal thrusters and rudders. It is shown in the figure that a peak in $P_{M}$ of approximately $180 \mathrm{~W}$ occurred during the sudden turn for both control schemes, where the thrusters were set to spin very fast.

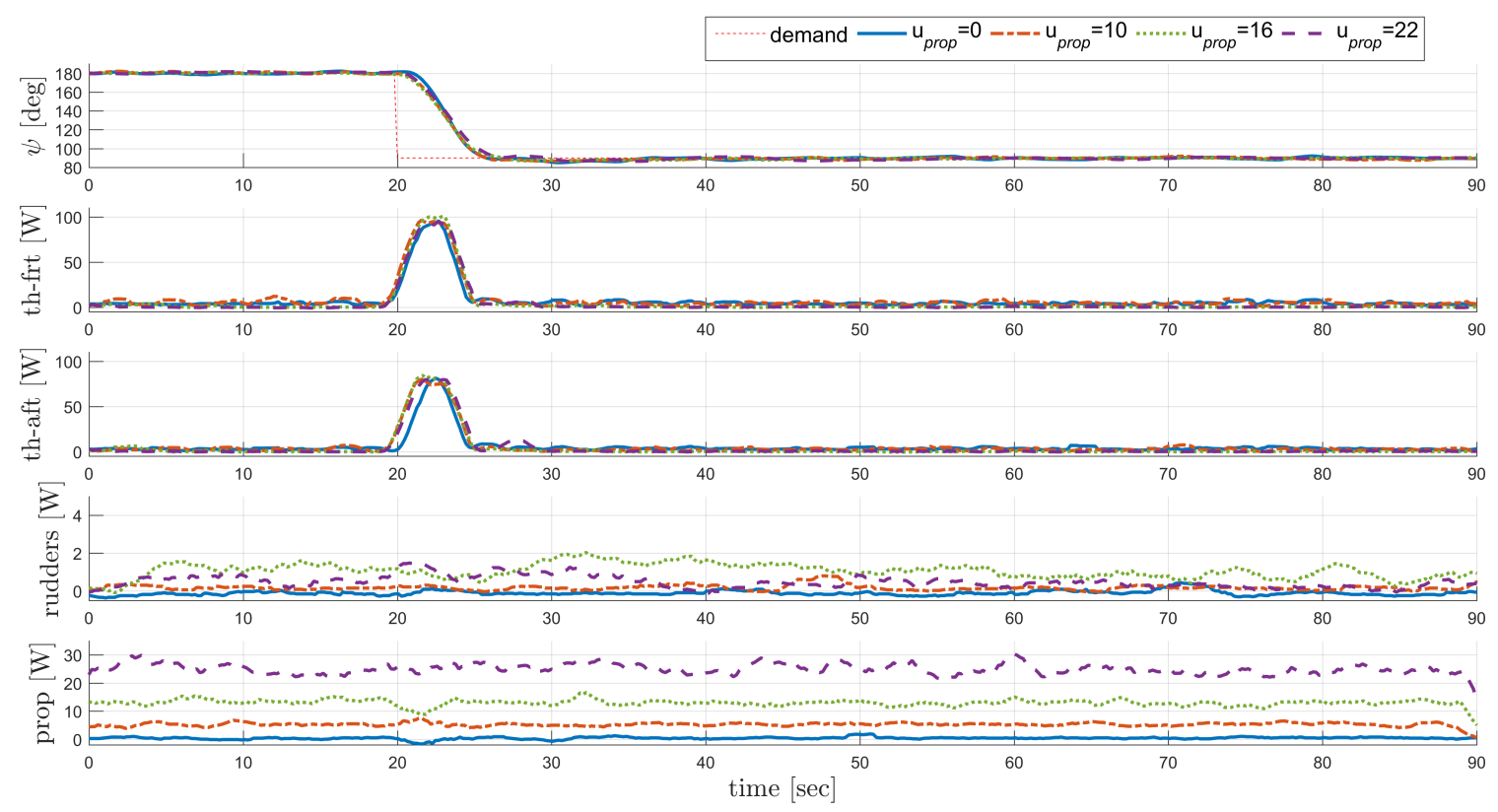

(a) SMC

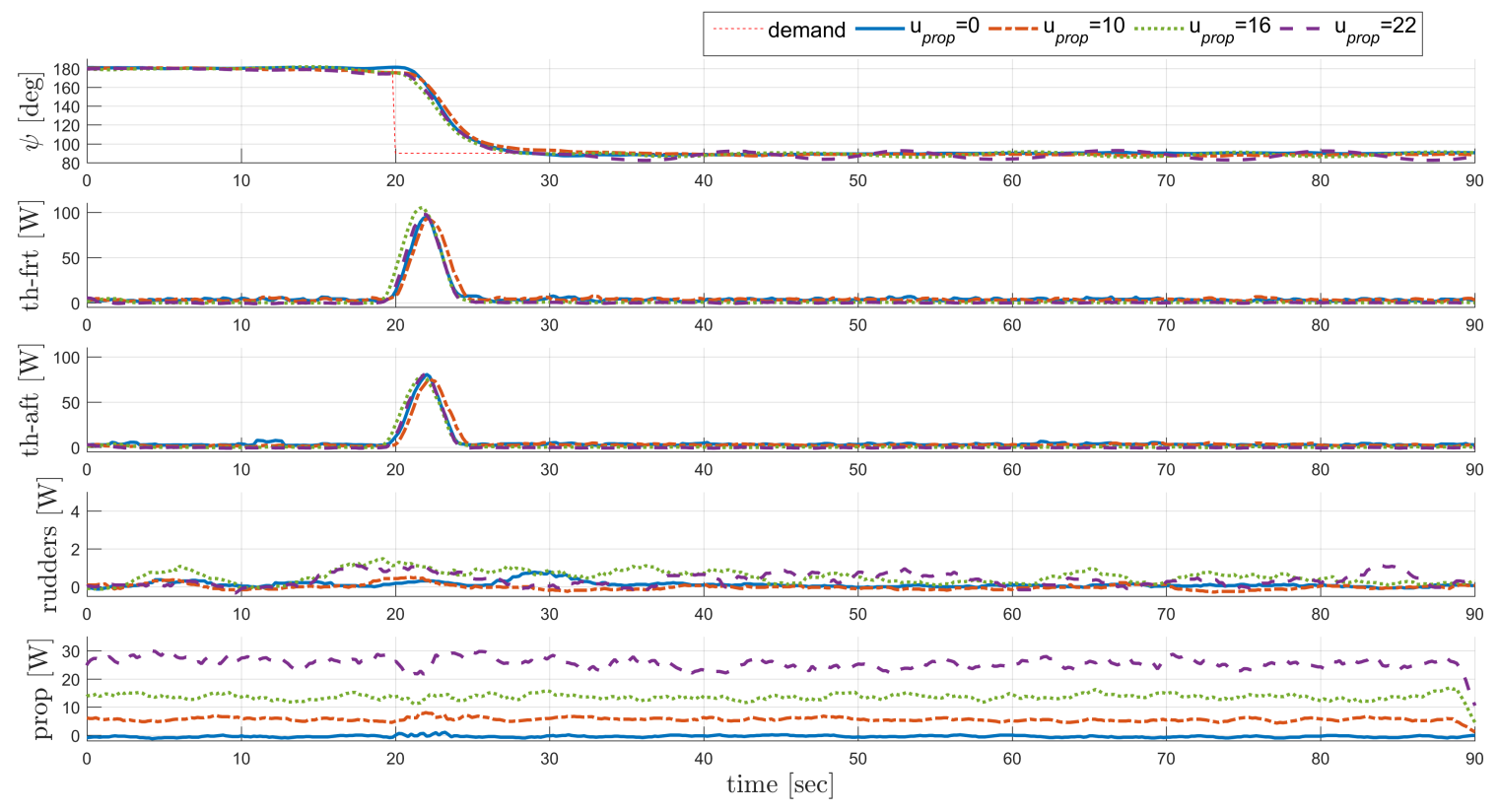

(b) P-D

Figure 17: Comparison on power consumption profile from two different heading control schemes. 
Manoeuvring energy, $E_{M}$, during the sudden turn for SMC and P-D control schemes are tabulated in tables 3 and 4 respectively; it is determined by integrating $P_{M}$ over time from the initiation of the heading demand change till reaching a steady state on the new heading - this is equivalent to an interval of between $19^{\text {th }}$ and $27^{\text {th }}$ second. Although the two approaches were using the same control allocation technique, the SMC scheme consumed more energy during a sudden turn when compared to the P-D scheme. Particularly, in the high-speed case, it consumed approximately $50 \%$ more energy. This is due to a characteristic of the SMC control law that generates more intense control actions to suppress the external disturbances and highly coupled surge-yaw dynamics of the vehicle. For this reason, as confirmed by section 7.1, the SMC control scheme yields consistent control performance regardless of the forward speeds.

Table 3: Energy consumption during turn (SMC)

\begin{tabular}{|c|c|c|c|c|c|}
\hline \multirow[b]{2}{*}{$\mathrm{u}_{\text {prop }}$} & \multirow{2}{*}{$\begin{array}{c}u \\
{[\mathrm{~m} / \mathrm{s}]}\end{array}$} & \multicolumn{3}{|c|}{ energy consumption $[\mathrm{J}]$} & \multirow{2}{*}{$E_{M}[\mathrm{~J}]$} \\
\hline & & th-frt & th-aft & fins & \\
\hline 0 & 0.00 & 321.2381 & 233.044 & 0.731 & 555.01 \\
\hline 10 & 0.42 & 374.9692 & 299.0506 & 1.2736 & 675.29 \\
\hline 16 & 0.82 & 364.7894 & 308.9138 & 7.9218 & 681.63 \\
\hline 22 & 1.03 & 358.5513 & 309.609 & 8.9951 & 677.16 \\
\hline
\end{tabular}

Table 4: Energy consumption during turn (P-D)

\begin{tabular}{|r|r|r|c|c|c|}
\hline \multirow{2}{*}{$\mathrm{u}_{\text {prop }}$} & \multirow{2}{*}{$\begin{array}{c}u \\
{[\mathrm{~m} / \mathrm{s}]}\end{array}$} & \multicolumn{2}{|c|}{ energy consumption $[\mathrm{J}]$} & \multirow{2}{*}{$E_{M}[\mathrm{~J}]$} \\
\hline \hline 0 & 0.00 & 247.318 & 202.7591 & 1.9304 & 452.01 \\
\hline 10 & 0.42 & 263.1534 & 201.9175 & 1.8432 & 466.91 \\
\hline 16 & 0.82 & 291.8407 & 213.8889 & 8.8726 & 514.60 \\
\hline 22 & 1.03 & 247.9194 & 194.6523 & 6.4437 & 449.02 \\
\hline
\end{tabular}

Power consumption, while AUV was maintaining a constant heading at different speeds, is obtained from the same experiment. These results correspond to SMC approach and P-D approach are tabulated in tables 5 and 6 , respectively, and are compared in figure 18. To maintain a constant heading at zero speed, manoeuvring power $\left(P_{M}\right)$ of $7.69 \mathrm{~W}$ and $6.50 \mathrm{~W}$ were required for SMC and P-D approaches, respectively. The $P_{M}$ for both approaches dropped to below $0.4 \mathrm{~W}$ for the high-speed case because only the rudder operation alone was sufficient for the heading tracking. This suggests that, during the steady state, the SMC scheme consumed at most $24 \%$ more energy than the P-D scheme, but this came with much more consistent and robust heading tracking performance over a wide range of operating speeds.

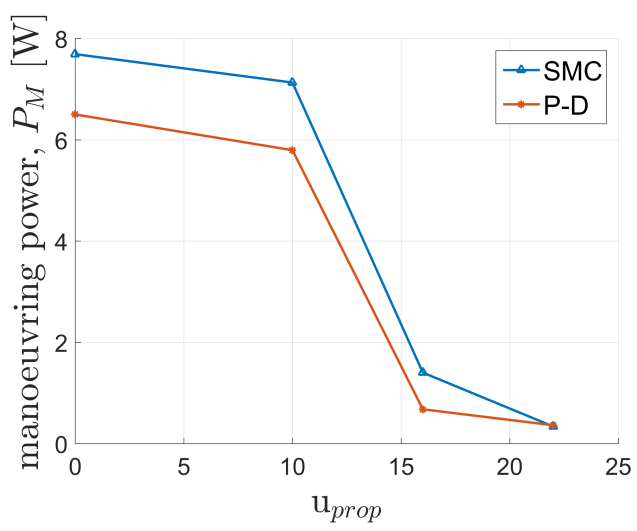

Figure 18: The SMC approach requires more manoeuvring power to maintain a constant heading than the P-D approach as a trade-off between efficiency and robust control performance. 
Table 5: Power consumption while maintaining a constant heading (SMC)

\begin{tabular}{|r|r|r|r|r|r|}
\hline \multirow{2}{*}{$\mathrm{u}_{\text {prop }}$} & \multirow{2}{*}{$\begin{array}{c}u \\
{[\mathrm{~m} / \mathrm{s}]}\end{array}$} & \multicolumn{3}{|c|}{ power consumption [W] } & \multirow{2}{*}{$P_{M}[\mathrm{~W}]$} \\
\cline { 3 - 5 } & & th-frt & th-aft & fins & \\
\hline \hline 0 & 0.00 & 4.27 & 3.42 & 0.00 & 7.69 \\
\hline 10 & 0.42 & 4.26 & 2.66 & 0.20 & 7.13 \\
\hline 16 & 0.82 & 0.33 & 0.06 & 1.01 & 1.40 \\
\hline 22 & 1.03 & 0.00 & 0.00 & 0.34 & 0.34 \\
\hline
\end{tabular}

Table 6: Power consumption while maintaining a constant heading (P-D)

\begin{tabular}{|r|r|r|r|r|r|}
\hline \multirow{2}{*}{$\mathrm{u}_{\text {prop }}$} & $u$ & \multicolumn{2}{|c|}{ power consumption $[\mathrm{W}]$} & \multirow{2}{*}{$P_{M}[\mathrm{~W}]$} \\
\cline { 3 - 5 } & {$[\mathrm{m} / \mathrm{s}]$} & th-frt & th-aft & \multicolumn{1}{|c|}{ fins } & \\
\hline \hline 0 & 0.00 & 3.43 & 3.07 & 0.00 & 6.50 \\
\hline 10 & 0.42 & 3.25 & 2.53 & 0.01 & 5.79 \\
\hline 16 & 0.82 & 0.16 & 0.05 & 0.47 & 0.68 \\
\hline 22 & 1.03 & 0.00 & 0.00 & 0.36 & 0.36 \\
\hline
\end{tabular}

\subsection{Path following missions}

This aims to demonstrate the performance of the proposed sliding mode heading control system in a practical mission, where the control module collaborates with a line-of-sight (LOS) guidance module based on Fossen et al. (2003). The LOS guidance concept is illustrated in figure 19. The AUV is considered to be enclosed by an invisible circle with a constant radius called LOS distance, $L_{l o s}$. An interception between the circle and the path section towards the target waypoint is denoted as LOS position, $p_{l o s}$. By having the AUV heading, $\psi$, converges to a LOS angle, $\psi_{l o s}$, it is guaranteed that the AUV converges to and navigates along the path towards the target waypoint. Once the AUV appears within a radius of acceptance, $R_{w p}$, it moves onto tracking the next waypoint in the waypoint sequence. The guidance parameters used during the mission are are: $L_{l o s}=10 \mathrm{~m} ; R_{w p}=5 \mathrm{~m}$.

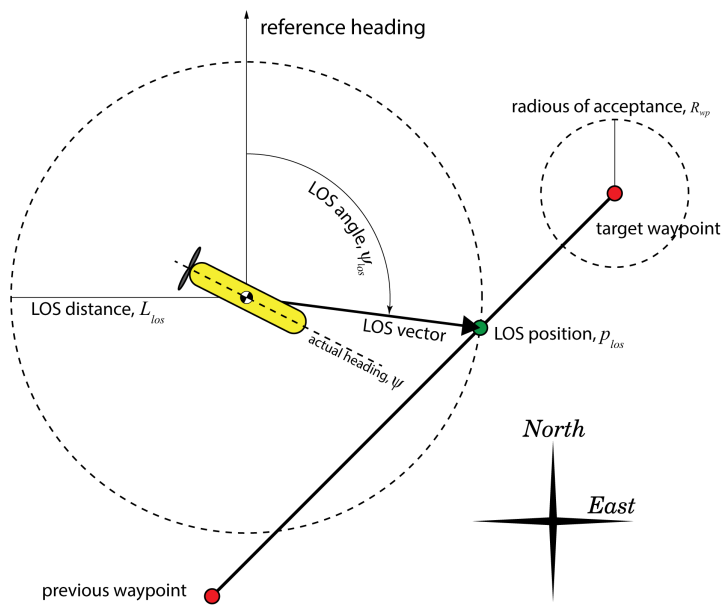

Figure 19: Line-of-sight guidance law.

The path following system was verified using straight-line and S-shaped paths. These paths are described as a set of waypoints where the coordinates are given in appendix $\mathrm{C}$.

The straight-line path was used to demonstrate a direction keeping performance at high-speed operation for both SMC and P-D scheme. In this scenario, the vehicle was programmed to transit with a constant 
propeller demand of $\mathrm{u}_{\text {prop }}=22$, starting from the launch location and visiting the prescribed waypoints in the following sequence: $\mathrm{M} \rightarrow \mathrm{B} \rightarrow \mathrm{A} \rightarrow \mathrm{B}$.

Comparing figure 20a with figure 20b, it is shown that the SMC scheme yielded a trace with a much tighter cross-track error band when compared with the P-D scheme. System response for the straight-line path following is presented in figures 20c and 20d: both approaches mainly used the rudders for maintaining the direction when tracking along the straight path, and the thrusters were used only during sharp turns. The SMC approach yielded slightly less variation in the heading error, but this required more intense control actions as a trade-off.

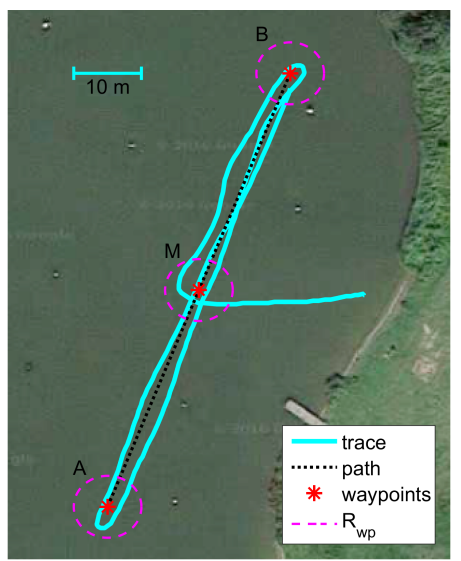

(a) trace: SMC
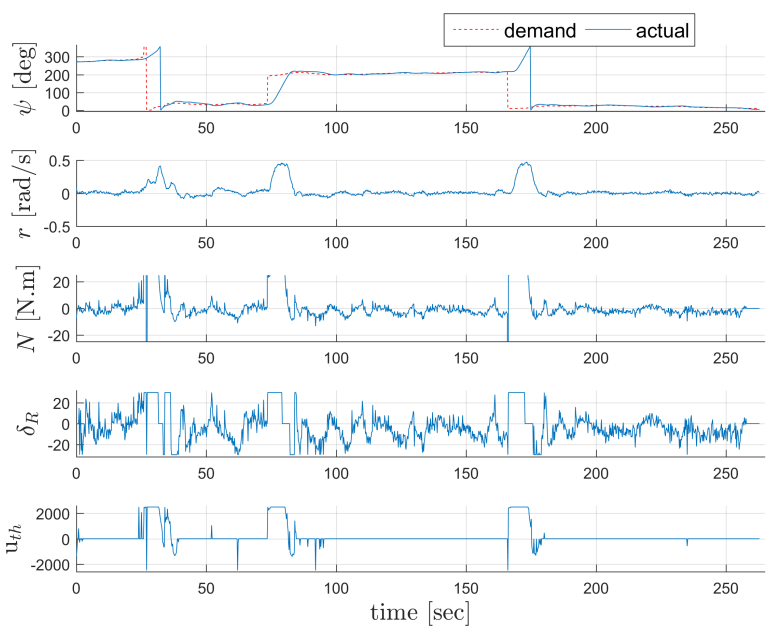

(c) response: SMC

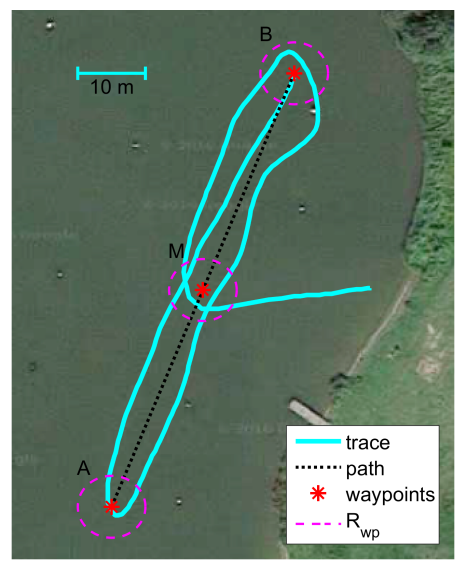

(b) trace: P-D
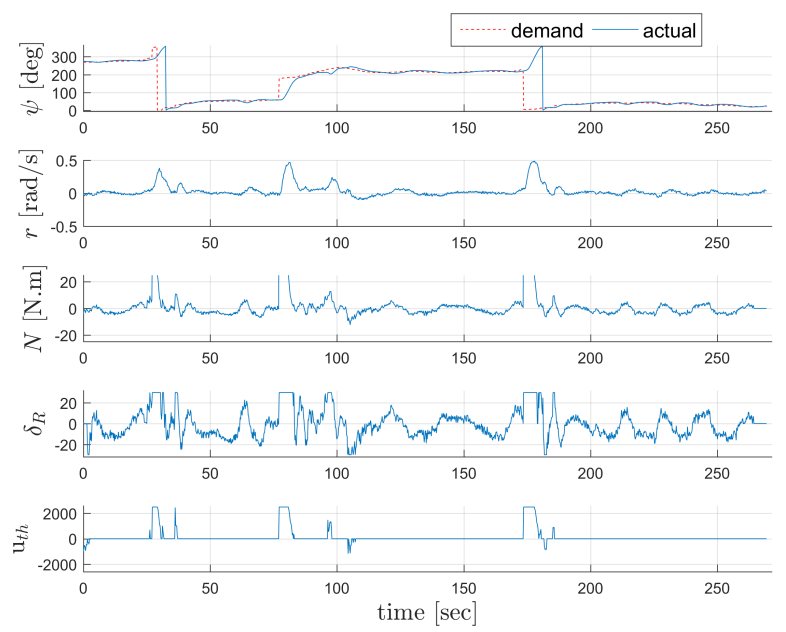

(d) response: P-D

Figure 20: Comparison on the straight-line path following between two control schemes $\left(\mathrm{u}_{\text {prop }}=22\right)$.

The S-shaped path was used for demonstrating the control system performance when following a curved path. Traces obtained from the experiment are given in figures 21a and $21 \mathrm{~b}$ for low-speed operation, and $22 \mathrm{a}$ and $22 \mathrm{~b}$ for high-speed operation. The difference in performance between SMC and P-D scheme is not obvious, for neither low- nor high-speed operations. However, the operating speeds significantly influence the outcomes. In the high-speed case, the AUV tended to go beyond the waypoint, whereas slow-speed operation provided more time for the AUV to aim for the next waypoint, yielding a better path tracking precision.

The waypoint overshoot at high speeds is partly because the existing GPS module gives a position update 
at only $1 \mathrm{~Hz}$ with an error of $\pm 2 \mathrm{~m}$. One possible way to achieve a higher position update rate is to fuse the estimated position from a dead reckoning algorithm with the GPS reading (Caron et al., 2006). The overshoot is also predicted to vary with the choice of $L_{l o s}$ and $R_{w p}$. The smaller values of these parameters would give a better path tracking precision. However, too small $R_{w p}$ would be difficult to reach, and too small $L_{l o s}$ would cause the AUV to oscillate along the path, especially, with the quality of the position information available at present.

By using the depth control system presented earlier (Tanakitkorn et al., 2016) in conjunction with the heading control system presented in this paper, the Delphin2 AUV is capable of navigating in 3D space. However, for fully-submerged operation, a position update will be obtained using alternative approaches instead of the GPS. These are, for example, acoustic positioning systems (Vickery, 1998) or simultaneous localisation and mapping (SLAM) techniques (Fairfield et al., 2007; Saeedi et al., 2016).

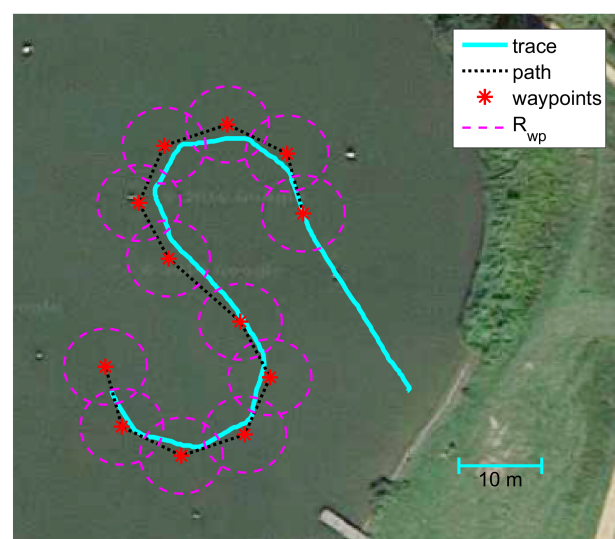

(a) trace: SMC
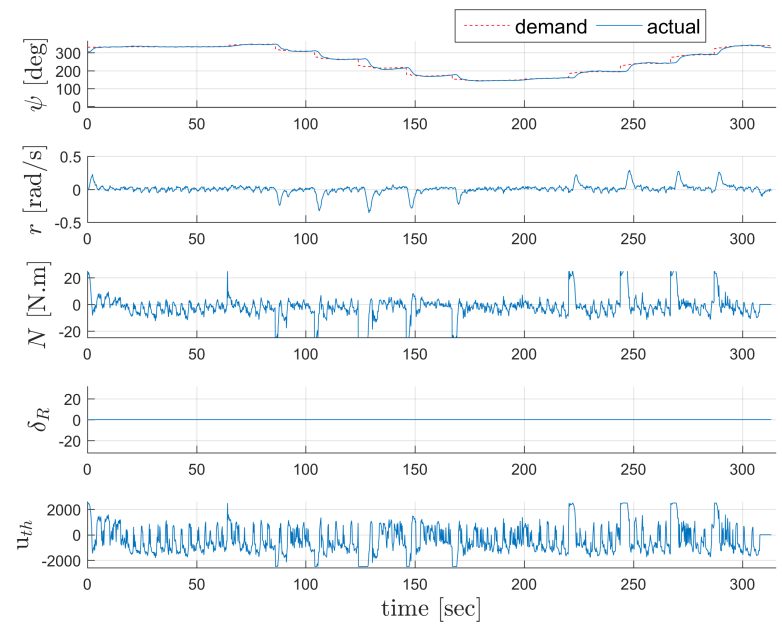

(c) response: SMC

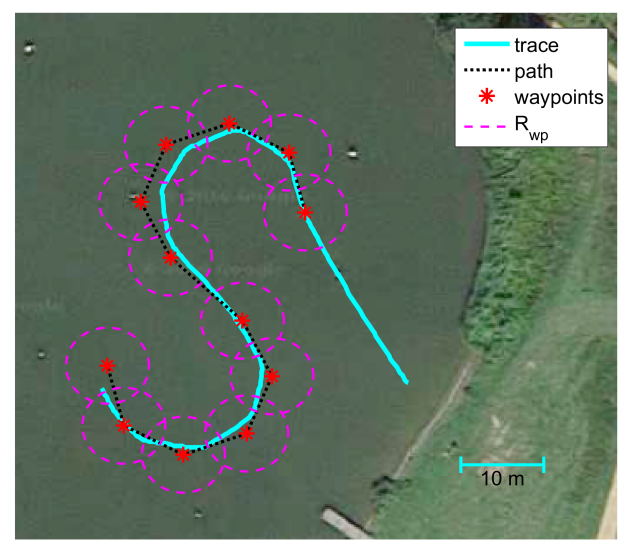

(b) trace: P-D
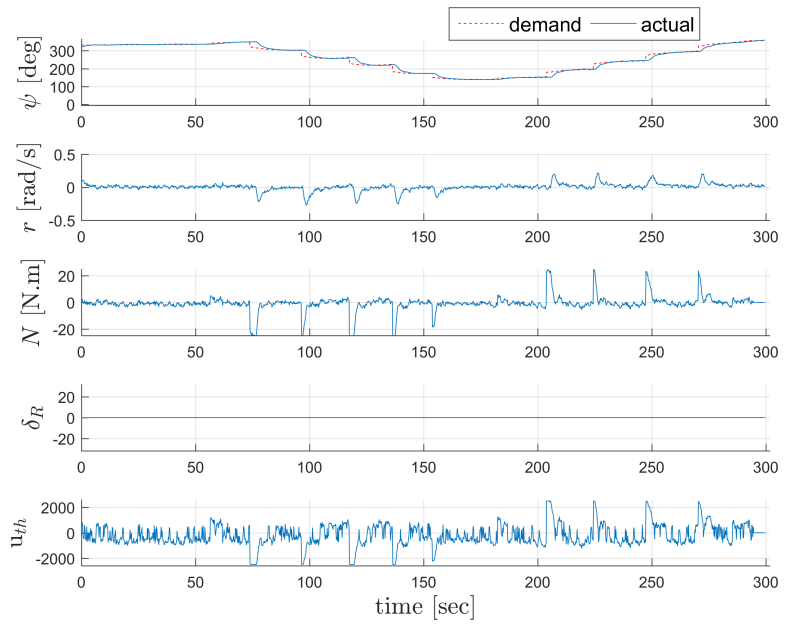

(d) response: P-D

Figure 21: Comparison on S-shaped path following between two control schemes $\left(\mathrm{u}_{\text {prop }}=10\right)$. 


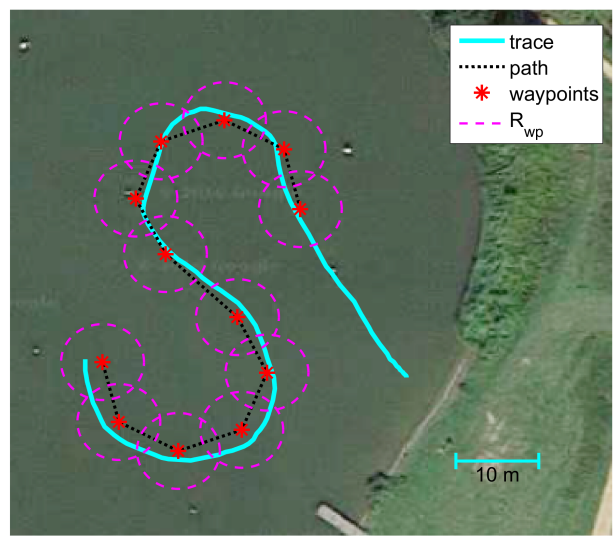

(a) trace: SMC
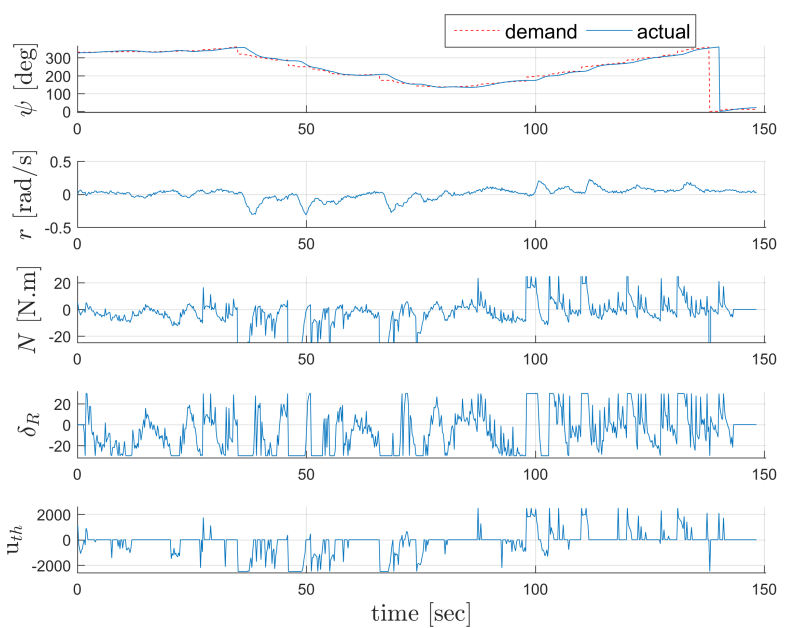

(c) response: SMC

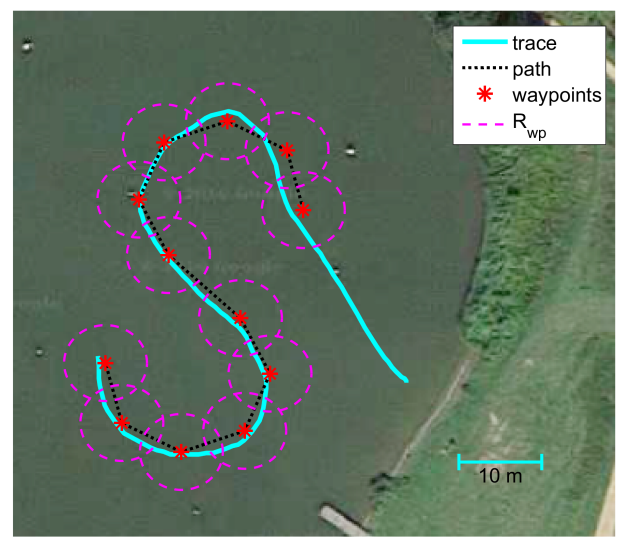

(b) trace: P-D
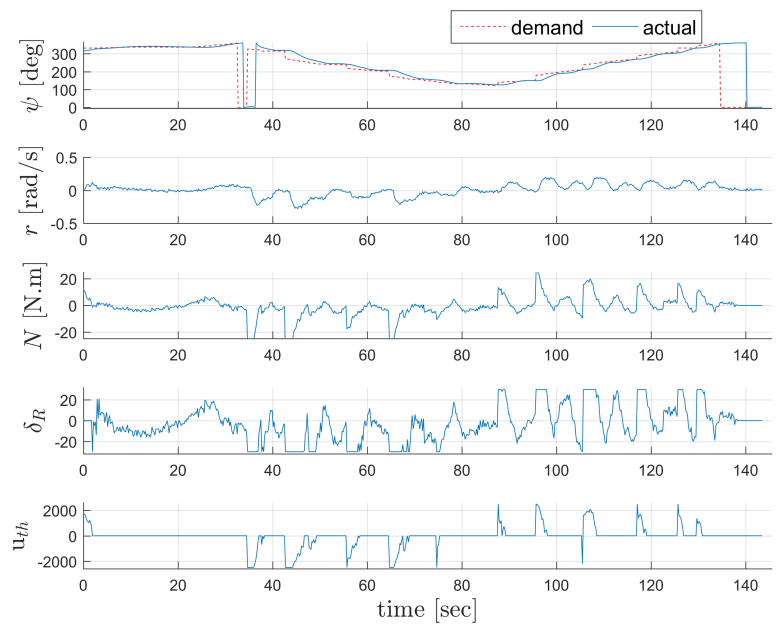

(d) response: P-D

Figure 22: Comparison on S-shaped path following between two control schemes $\left(\mathrm{u}_{\text {prop }}=22\right)$.

\section{Conclusions}

This paper presents a systematic approach to design a sliding mode heading control system from the statespace model of the decoupled yaw dynamics. The control system consists of two parts: 1) the control law generates a control demand regarding the required yaw moment that is sufficient for heading tracking, 2) the daisy-chaining technique is then employed to effectively allocate the necessary moment between horizontal thrusters and rudders. This cascade control structure allows the presented control system to be applied to any vehicle straightforwardly. However, the control allocation part may need to be modified in according to the actuator configuration of that vehicle.

A novel sliding function, namely sSTA, is presented and compared to a set of switching function candidates. Simulation results show that sSTA does improve the chattering problem, yielding a much smoother control response. Also, it can better tolerate an external disturbance when compared to other switching function candidates. Simulation studies on parameter uncertainties are presented: the key model parameters involved in the control section are individually varied between $\pm 90 \%$ of the estimated values used in the simulation. The results show that the control system can tolerate a broad range of parameter variations. However, the moment of inertia $\left(I_{z z}\right)$ and yaw damping coefficients $\left(N_{r}\right.$ and $\left.N_{|r| r}\right)$ highly affect the transient response of the controller because they are directly related to the yaw dynamics. Therefore, great care must, therefore, 
be taken when estimating these parameters to minimise negative effects of the model mismatch.

Lake trials are presented, demonstrating good heading tracking performance when experiencing an external disturbance in a practical environment. With the proposed SMC scheme, the AUV takes less than 6 sec to gain a 90 deg heading change without overshoot, and the heading error is maintained within \pm 2 deg during a steady state, yielding an excellent performance regardless of the forward speeds. As supported by the simulation studies in this work, the SMC can tolerate to an extreme level of the parameter uncertainties and maintain a stable heading tracking response over a possible range of the parameter values that may change when the AUV is fully-submerged. The presented SMC also offers a smooth control response which is one of the key factors that contributes to good quality sensor data, e.g., a seafloor map from a sidescan sonar.

When comparing to the P-D control scheme implemented on the same vehicle, the proposed SMC scheme shows superior performance that is more consistent regardless of the forward speeds. This highlights that the SMC approach is more suitable for the AUV heading control problem that is highly nonlinear. Energy consumption during sudden turn tests was recorded to analyse operational efficiency. The results suggest that using the SMC scheme causes the vehicle to spend at most $24 \%$ more manoeuvring energy than using the P-D scheme while maintaining a constant heading. This is due to more intense control actions that are required as a trade-off for the robustness property of SMC scheme. Since the consistent performance far outweighs the concern on energy consumption, SMC is considered more suitable for AUVs that have to operate over the range of forward speeds.

Path following missions are proposed to demonstrate the applicability of the control system in realistic operation, where the control module is collaborating with the line-of-sight guidance module. The results show that the SMC scheme yields a smaller cross-track error band than the P-D scheme during a straightline path following because of its relatively more robust heading tracking performance. When tracking the S-shaped path, both control schemes produce a very subtle difference in performance. However, the resulting traces from both schemes tend to slightly overshoot from the given path, especially in high-speed operation. This is due to an unreliable position information that is provided by the GPS module at $1 \mathrm{~Hz}$ with an error of $\pm 2 \mathrm{~m}$. Despite this unreliable position update, the two control schemes successfully manage to visit all the prescribed waypoints and complete the path.

\section{Acknowledgments}

This research is sponsored by the Faculty of International Maritime Studies, Kasetsart University, SriRacha Campus, Thailand. The gratitude is also extended to Sophia Schillai and Prin Kanyoo for their great support during the vehicle preparation and experiments.

\section{A P-D control law}

The control scheme used in this work slightly differs from the conventional proportional-derivative (PD) scheme: 1) a derivative term is placed in a feedback path instead of the forward path, 2) an actual state of the system is fed to the derivative term instead of the tracking error. (See figure 23.) Thus, the derivative term is not affected by a sudden change in the desired state, i.e. avoiding the setpoint kick phenomenon (Ogata, 2009). By doing so, the derivative gains must have a negative value; hence it is commonly referred to as a P-D scheme.

The P-D control law, that determines the generalised yaw moment for the heading control, is given by the form:

$$
N_{t o t}=K_{P, \psi} \tilde{\psi}+K_{D, \psi} \dot{\psi}
$$

where gains are tuned at zero-speed operation based on Ziegler-Nichols method (Ogata, 2009). 


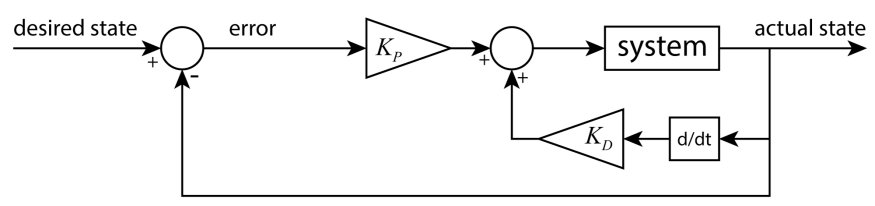

Figure 23: P-D control scheme.

This generalised yaw moment is then distributed between thrusters and rudders using a daisy-chaining control allocation technique, see section 3.3.

\section{B Model parameters}

\begin{tabular}{lll}
\hline \hline Parameter & Value & Unit \\
\hline$m$ & 79.40 & $\mathrm{~kg}$ \\
$\nabla$ & 0.08 & $\mathrm{~m}{ }^{3}$ \\
$L_{A U V}$ & 1.96 & $\mathrm{~m}$ \\
$I_{z z}$ & 35 & $\mathrm{~kg} \cdot \mathrm{m}^{2}$ \\
$r_{b}$ & {$[0,0,0]$} & $\mathrm{m}$ \\
$r_{g}$ & {$[0,0,0.06]$} & $\mathrm{m}$ \\
$X_{\dot{u}}$ & -2.4 & $\mathrm{~kg}$ \\
$Y_{\dot{v}}$ & -65.5 & $\mathrm{~kg}$ \\
$N_{\dot{r}}$ & -14.17 & $\mathrm{~kg} \cdot \mathrm{m}^{2} / \mathrm{rad}$ \\
$Y_{v}$ & $0.5 \rho L_{A U V}^{2} u(-28.5 \mathrm{e}-3)$ & $\mathrm{kg} / \mathrm{s}$ \\
$Y_{r}$ & $0.5 \rho L_{A U V}^{3} u(12.6 \mathrm{e}-3)$ & $\mathrm{kg} \cdot \mathrm{m} / \mathrm{rad} / \mathrm{s}$ \\
$N_{v}$ & $0.5 \rho L_{A U V}^{3} u(4.5 \mathrm{e}-3)$ & $\mathrm{kg} \cdot \mathrm{m} / \mathrm{s}$ \\
$N_{r}$ & $0.5 \rho L_{A U V}^{4} u(-5.3 \mathrm{e}-3)$ & $\mathrm{kg} \cdot \mathrm{m}^{2} / \mathrm{rad} / \mathrm{s}$ \\
$X_{|u| u}$ & -2.79 & $\mathrm{~kg} / \mathrm{m}$ \\
$Y_{|v| v}$ & -183.22 & $\mathrm{~kg} / \mathrm{m}$ \\
$Y_{|r| r}$ & 0 & $\mathrm{~kg} \cdot \mathrm{m} / \mathrm{rad}^{2}$ \\
$N_{|v| v}$ & 59 & $\mathrm{~kg}$ \\
$N_{|r| r}$ & -82 & $\mathrm{~kg} \cdot \mathrm{m}^{2} / \mathrm{rad}^{2}$ \\
$K_{T, t h}$ & $1.2870 \mathrm{e}-4$ & - \\
$D_{t h}$ & 0.07 & $\mathrm{~m}$ \\
$L_{h}$ & 1.25 & $\mathrm{~m}$ \\
$N_{u u \delta_{R}}$ & 0.3254 & $\mathrm{~kg} / \mathrm{deg}$ \\
\hline
\end{tabular}

\section{Path waypoints}

Two sets of waypoints are given in metre in relative to the reference point nearby the pier, see table 7 and 8.

Table 7: Waypoints for a straight-line path

\begin{tabular}{|l|l|l|l|}
\hline & $\mathrm{A}$ & $\mathrm{B}$ & $\mathrm{M}$ \\
\hline $\mathrm{X}$ & -28 & -1 & -14.5 \\
\hline $\mathrm{Y}$ & -20 & 50 & 15 \\
\hline
\end{tabular}


Table 8: Waypoints for an S-shaped path

\begin{tabular}{|l|l|l|l|l|l|l|l|l|l|l|l|l|}
\hline $\mathrm{X}$ & -3.8 & -5.7 & -12.9 & -20.6 & -23.6 & -20.0 & -11.4 & -7.8 & -10.8 & -18.5 & -25.7 & -27.6 \\
\hline $\mathrm{Y}$ & 36.2 & 44.1 & 47.9 & 45.1 & 37.6 & 30.3 & 22.0 & 14.7 & 7.1 & 4.3 & 8.1 & 16.0 \\
\hline
\end{tabular}

\section{Controller gains}

Table 9: Gains for P-D control scheme

\begin{tabular}{|l|l|}
\hline$K_{P, \psi}$ & $K_{D, \psi}$ \\
\hline \hline 0.8 & -1.2 \\
\hline
\end{tabular}

Table 10: Gains for SMC control scheme

\begin{tabular}{|l|r|c|r|r|r|}
\hline & $h_{1}$ & \multicolumn{1}{|c|}{$h_{2}$} & $K_{s w, 1}$ & $K_{s w, 2}$ & $\sigma^{*}$ \\
\hline \hline sign & 1 & 0.01 & 0.4 & - & - \\
\hline con & 1 & 0.01 & 0.4 & - & 0.2 \\
\hline STA & 1 & 0.01 & 0.6 & 0.15 & - \\
\hline sSTA & 1 & 0.01 & 0.5 & 0.15 & 0.2 \\
\hline
\end{tabular}

\section{References}

Allen, B., Stokey, R., Austin, T., Forrester, N., Goldsborough, R., Purcell, M., and Alt, C. V. (1997). REMUS: a small, low cost AUV; system description, field trials and performance results. Oceans ' 97. MTS/IEEE Conference Proceedings, 2.

Burcher, R. and Rydill, L. J. (1995). Concepts in Submarine Design. Cambridge Ocean Technology Series. Cambridge University Press.

Caron, F., Duflos, E., Pomorski, D., and Vanheeghe, P. (2006). GPS/IMU data fusion using multisensor Kalman filtering: Introduction of contextual aspects. Information Fusion, 7(2):221-230.

Conte, G., De Capua, G., and Scaradozzi, D. (2016). Designing the NGC system of a small ASV for tracking underwater targets. Robotics and Autonomous Systems, 76:46-57.

DeCarlo, R., Zak, S., and Matthews, G. (1988). Variable Structure Control of Nonlinear Multivariable Systems : A Tutorial. In Proceedings of the IEEE, 76(3):212-232.

Dunbabin, M., Roberts, J., Usher, K., Winstanley, G., and Corke, P. (2005). A Hybrid AUV Design for Shallow Water Reef Navigation. In Proceedings of the 2005 IEEE International Conference on Robotics and Automation, pages 2105-2110. IEEE.

Fairfield, N., Kantor, G., and Wettergreen, D. (2007). RealTime SLAM with Octree Evidence Grids for Exploration in Underwater Tunnels. Journal of Field Robotics, 24:3-21.

Fossen, T. I. (2011). Handbook of Marine Craft Hydrodynamics and Motion Control. John Wiley \& Sons, Ltd, Chichester, UK.

Fossen, T. I., Breivik, M., and Skjetne, R. (2003). Line-of-sight path following of underactuated marine craft. Proceedings of the 6th IFAC MCMC, Girona, Spain, pages 244-249.

Fossen, T. I. and Johansen, T. (2006). A Survey of Control Allocation Methods for Ships and Underwater Vehicles. In 2006 14th Mediterranean Conference on Control and Automation, pages 1-6. IEEE.

Han, Y. and Liu, X. (2016). Continuous higher-order sliding mode control with time-varying gain for a class of uncertain nonlinear systems. ISA Transactions, pages 1-9.

Hanger, M., Johansen, T. a., Mykland, G. K., and Skullestad, A. (2011). Dynamic model predictive control allocation using CVXGEN. In 2011 9th IEEE International Conference on Control and Automation (ICCA), pages 417-422. IEEE. 
Jantapremjit, P. and Wilson, P. A. (2007). Optimal Control and Guidance for Homing and Docking Tasks using an Autonomous Underwater Vehicle. In 2007 International Conference on Mechatronics and Automation, pages 243-248. IEEE.

Jantapremjit, P. and Wilson, P. A. (2008). Guidance-Control Based Path Following for Homing and Docking using an Autonomous Underwater Vehicle. OCEANS 2008 - MTS/IEEE Kobe Techno-Ocean, (1):1-6.

Jin, S., Kim, J., Kim, J., and Seo, T. (2014). Six-Degree-of-Freedom Hovering Control of an Underwater Robotic Platform With Four Tilting Thrusters via Selective Switching Control. In Mechatronics, IEEE/ASME Transactions on, vol.PP, no.99, pages 1-9. IEEE.

Kim, M., Joe, H., Kim, J., and Yu, S.-c. (2015). Integral sliding mode controller for precise manoeuvring of autonomous underwater vehicle in the presence of unknown environmental disturbances. International Journal of Control, 88(10):2055-2065.

Kokegei, M., He, F., and Sammut, K. (2011). Fully Coupled 6 Degree-of-Freedom Control of an OverActuated Autonomous Underwater Vehicle. In Cruz, N., editor, Autonomous Underwater Vehicles, pages 147-170. InTech.

Lea, R. K. (1998). A comparative study by simulation and experimentation of control techniques for autonomous underwater flight vehicles. PhD thesis, University of Southampton.

Lekkas, A. M. (2014). Guidance and Path-Planning Systems for Autonomous Vehicles. PhD thesis, Norwegian University of Science and Technology.

Levant, A. (2007). Principles of 2-sliding mode design. Automatica, 43(4):576-586.

Levant, A., Pridor, A., Gitizadeh, R., Yaesh, I., and Ben-Asher, J. Z. (2000). Aircraft Pitch Control via Second-Order Sliding Technique. Journal of Guidance, Control, and Dynamics, 23(4):586-594.

Li, B. and Su, T.-c. (2016). Nonlinear heading control of an autonomous underwater vehicle with internal actuators. Ocean Engineering, 125:103-112.

Liu, J., Furlong, M. E., Palmer, A., Phillips, A. B., Turnock, S. R., and Sharkh, S. M. (2009). Design and Control of a Flight-Style AUV with Hovering Capability. In Proceedings of the International Symposium on Unmanned Untethered Submersible Technology (UUST 2009), pages 1-9, Durham NH, USA. Autonomous Undersea Systems Institute (AUSI).

Marco, D. and Healey, A. (2000). Current developments in underwater vehicle control and navigation: the NPS ARIES AUV. OCEANS 2000 MTS/IEEE Conference and Exhibition. Conference Proceedings (Cat. No.00CH37158), 2.

McPhail, S. (2009). Autosub6000: A Deep Diving Long Range AUV. Journal of Bionic Engineering, $6(1): 55-62$.

Morari, M. (2012). Sliding Modes after the First Decade of the 21st Century, volume 412 of Lecture Notes in Control and Information Sciences. Springer Berlin Heidelberg, Berlin, Heidelberg.

Naeem, W., Sutton, R., Chudley, J., Dalgleish, F. R., and Tetlow, S. (2005). An online genetic algorithm based model predictive control autopilot design with experimental verification. International Journal of Control, 78(14):1076-1090.

Ogata, K. (2009). Modern Control Engineering. Prentice Hall, 5 edition.

Oppenheimer, M. W., Doman, D. B., and Bolender, M. A. (2006). Control Allocation for Over-actuated Systems. In 2006 14th Mediterranean Conference on Control and Automation, pages 1-6. IEEE.

Ouyang, P., Pano, V., and Hu, Y. (2015). Position domain PD sliding mode control for contour tracking. In 2015 IEEE International Conference on Advanced Intelligent Mechatronics (AIM), pages 1020-1025. IEEE.

Packard, G. E., Stokey, R., Christenson, R., Jaffre, F., Purcell, M., and Littlefield, R. (2010). Hull inspection and confined area search capabilities of REMUS autonomous underwater vehicle. Oceans 2010, pages 1-4.

Perruquetti, W. and Barbot, J. (2002). Sliding mode control in engineering. CRC Press. 
Phillips, A. B., Steenson, L. V., and Liu, J. (2013). Delphin2: An over actuated autonomous underwater vehicle for manoeuvring research. Transactions of the Royal Institution of Naval Architects, Part A Engineering, 151(0092-8674):1-11.

Ribas, D., Ridao, P., Turetta, A., Melchiorri, C., Palli, G., Fernandez, J. J., and Sanz, P. J. (2015). I-AUV Mechatronics Integration for the TRIDENT FP7 Project. IEEE/ASME Transactions on Mechatronics, 20(5):2583-2592.

Saeedi, S., Trentini, M., Seto, M., and Li, H. (2016). Multiple-Robot Simultaneous Localization and Mapping: A Review. Journal of Field Robotics, 33(1):3-46.

Shi, J., Liu, H., and Bajcinca, N. (2008). Robust control of robotic manipulators based on integral sliding mode. International Journal of Control, 81(10):1537-1548.

Shtessel, Y., Edwards, C., Fridman, L., and Levant, A. (2014). Sliding Mode Control and Observation.

Silvestre, C. and Pascoal, A. (2004). Control of the INFANTE AUV using gain scheduled static output feedback. Control Engineering Practice, 12(12):1501-1509.

Slotine, J. E. and Li, W. (1991). Applied nonlinear control. Prentice Hall.

Spurgeon, S. (2014). Sliding mode control: a tutorial. 2014 European Control Conference (ECC), (1):22722277.

Steenson, L. V. (2013). Experimentally Verified Model Predictive Control of a Hover-Capable AUV. PhD thesis, University of Southampton.

Steenson, L. V., Phillips, A. B., Furlong, M. E., Rogers, E., and Turnock, S. R. (2011a). Maneuvering of an over-actuated autonomous underwater vehicle using both through-body tunnel thrusters and control surfaces. 17th International Undersea Untethered Submersible Technology Conference.

Steenson, L. V., Phillips, A. B., Furlong, M. E., Rogers, E., and Turnock, S. R. (2011b). The performance of vertical tunnel thrusters on an autonomous underwater vehicle operating near the free surface in waves. In Second International Symposium on Marine Propulsors, pages 1-8.

Steenson, L. V., Turnock, S. R., Phillips, A. B., Harris, C., M.E. Furlong, Rogers, E., Wang, L., Bodles, K., D.W. Evans, Furlong, M. E., Rogers, E., Wang, L., Bodles, K., and Evans, D. W. (2014). Model predictive control of a hybrid autonomous underwater vehicle with experimental verification. Proceedings of the Institution of Mechanical Engineers, Part M: Journal of Engineering for the Maritime Environment, 228(2):166-179.

Tanakitkorn, K., Wilson, P. A., Turnock, S. R., and Phillips, A. B. (2016). Depth Control for an OverActuated, Hover-Capable Autonomous Underwater Vehicle with Experimental Verification. Submitted to Mechatronics.

Utkin, V. (1977). Variable structure systems with sliding modes. IEEE Transactions on Automatic Control, $22(2): 212-222$.

Utkin, V., Guldner, J., and Shi, J. (2009). Sliding Mode Control in Electro-Mechanical Systems, Second Edition. CRC Press, 2009, 2 edition.

Vickery, K. (1998). Acoustic positioning systems. A practical overview of current systems. In Proceedings of the 1998 Workshop on Autonomous Underwater Vehicles (Cat. No.98CH36290), pages 5-17. Ieee.

Vuilmet, C. (2005). High order sliding mode control applied to a heavyweight torpedo. In Proceedings of 2005 IEEE Conference on Control Applications, 2005. CCA 2005., pages 61-66. IEEE.

Yang, R., Clement, B., Mansour, A., Li, H. J., and Li, M. (2015). Robust heading control and its application to Ciscrea Underwater Vehicle. In OCEANS 2015 - Genova, pages 1-6. IEEE.

Yoerger, D. and Slotine, J.-J. E. (1985). Robust trajectory control of underwater vehicles. IEEE Journal of Oceanic Engineering, 10(4):462-470. 